ARTICLE

\title{
Highly efficient and robust noble-metal free bifunctional water electrolysis catalyst achieved via complementary charge transfer
}

\author{
Nam Khen Oh${ }^{1,3}$, Jihyung Seo ${ }^{1,3}$, Sangjin Lee ${ }^{2,3}$, Hyung-Jin Kim² ${ }^{2}$ Ungsoo Kim¹, Junghyun Lee ${ }^{1}$ \\ Young-Kyu Han (1) ${ }^{2 \times}$ \& Hyesung Park ${ }^{1 凶}$
}

The operating principle of conventional water electrolysis using heterogenous catalysts has been primarily focused on the unidirectional charge transfer within the heterostructure. Herein, multidirectional charge transfer concept has been adopted within heterostructured catalysts to develop an efficient and robust bifunctional water electrolysis catalyst, which comprises perovskite oxides $\left(\mathrm{La}_{0.5} \mathrm{Sr}_{0.5} \mathrm{CoO}_{3-\delta} \mathrm{LSC}\right)$ and potassium ion-bonded $\mathrm{MoSe}_{2}$ $\left(\mathrm{K}-\mathrm{MoSe}_{2}\right)$. The complementary charge transfer from LSC and $\mathrm{K}$ to $\mathrm{MoSe}_{2}$ endows $\mathrm{MoSe}_{2}$ with the electron-rich surface and increased electrical conductivity, which improves the hydrogen evolution reaction (HER) kinetics. Excellent oxygen evolution reaction (OER) kinetics of $\mathrm{LSC} / \mathrm{K}-\mathrm{MoSe}_{2}$ is also achieved, surpassing that of the noble metal $\left(\mathrm{IrO}_{2}\right)$, attributed to the enhanced adsorption capability of surface-based oxygen intermediates of the heterostructure. Consequently, the water electrolysis efficiency of $\mathrm{LSC} / \mathrm{K}-\mathrm{MoSe}_{2}$ exceeds the performance of the state-of-the-art $\mathrm{Pt} / \mathrm{C} \| \mathrm{IrO} \mathrm{O}_{2}$ couple. Furthermore, $\mathrm{LSC} / \mathrm{K}-\mathrm{MoSe}_{2}$ exhibits remarkable chronopotentiometric stability over 2,500 h under a high current density of $100 \mathrm{~mA} \mathrm{~cm}^{-2}$.

\footnotetext{
${ }^{1}$ Department of Materials Science and Engineering, Perovtronics Research Center, Low Dimensional Carbon Materials Center, Ulsan National Institute of Science and Technology (UNIST), Ulsan, Republic of Korea. ${ }^{2}$ Department of Energy and Materials Engineering and Advanced Energy and Electronic Materials Research Center, Dongguk University-Seoul, Seoul, Republic of Korea. ${ }^{3}$ These authors contributed equally: Nam Khen Oh, Jihyung Seo, Sangjin Lee.

凶email: ykenergy@dongguk.edu; hspark@unist.ac.kr
} 
ydrogen is one of the cleanest and most sustainable energy sources that can provide high-energy-density fuel for electricity generation. Moreover, hydrogen production via electrocatalytic water splitting has been extensively explored recently ${ }^{1,2}$. Noble-metal-based $\mathrm{Pt} / \mathrm{Pd}$ and $\mathrm{RuO}_{2} / \mathrm{IrO}_{2}$ electrocatalysts have been widely utilized in the cathodic hydrogen evolution reaction (HER) and anodic oxygen evolution reaction (OER), respectively, for efficient hydrogen generation from water electrolysis. Although noble-metal-based electrocatalysts exhibit excellent water electrolysis performance, these catalysts typically suffer from poor stability and scarcity in alkaline water electrolysis systems ${ }^{3,4}$, which is undesirable in practical industrial applications. In addition, most noble-metal-based catalysts function optimally only for the half-cell reaction (either HER or OER) under specific electrolytic conditions ${ }^{5}$. Bifunctional catalysts that can be simultaneously used for both the cathode and anode reactions in the same electrolyte have been developed to improve water electrolysis performance and simplify the electrolysis system ${ }^{6,7}$. For bifunctional catalysts, the reaction kinetics and efficiency of the OER process can determine the reaction rate of overall water electrolysis due to its sluggish kinetics from the rigid double bond in $\mathrm{O}-\mathrm{O}$ and multi-proton-coupled electron transfer steps ${ }^{8,9}$. Therefore, achieving effective OER catalytic activity in bifunctional catalysts is critical in maximizing water electrolysis performance.

To strategically enhance the water electrolysis efficiency of the bifunctional catalyst, heterostructured catalyst configurations, including transition-metal-based nanocrystals (e.g., $\mathrm{Co}, \mathrm{Ni}, \mathrm{Fe}$, and $\mathrm{Cu}$ ) or noble-metal-based composites (e.g., $\mathrm{Pt}, \mathrm{Ag}, \mathrm{Pd}, \mathrm{Au}$, and $\mathrm{Rh}$ ), have often been investigated for their morphology control, compositional combination of the heterostructure, and foreign elemental doping ${ }^{10-14}$. Charge transfer within the heterostructured catalyst, which can modulate the electronic structure of the heterostructure and directly influence the Faradaic efficiency of the respective electrode ${ }^{15,16}$, is critical in improving the efficiency of overall water electrolysis for bifunctional catalysts. Thus far, most bifunctional heterostructure-based catalysts have primarily utilized unidirectional charge transfer effects between heterostructure components ${ }^{17,18}$, which can potentially limit an optimized electronic structure to achieve ideal HER and OER catalytic activities. Therefore, a different perspective on catalyst design is needed to effectively modulate the electronic structure of the catalyst such that its electrocatalytic activity is maximized in water electrolysis. Furthermore, most bifunctional catalysts demonstrate operational stability under relatively low current densities ${ }^{19-23}$. As the durability of the electrolytic catalyst can be severely degraded at high current densities, e.g., through the Ostwald ripening, aggregation, and detachment during the electrolytic reaction 24,25 , robust electrochemical stability of the bifunctional catalyst should also be achieved for industrial consideration in addition to the high efficiency of water electrolysis.

In the present work, we have developed a heterostructured catalyst comprising perovskite oxide $\left(\mathrm{La}_{0.5} \mathrm{Sr}_{0.5} \mathrm{CoO}_{3-\delta}, \mathrm{LSC}\right)$ and potassium ion-bonded molybdenum diselenide $\left(\mathrm{K}-\mathrm{MoSe}_{2}\right)$ as the bifunctional catalysts for overall water electrolysis. The semiconducting 2H-phase $\mathrm{MoSe}_{2}$ was moderately converted to metallic $1 \mathrm{~T}-\mathrm{MoSe}_{2}$ via charge transfer from potassium atoms during the potassium metal intercalation process $^{26}$. In a previous report, Park et al. reported a heterostructured water electrolysis catalyst structure comprising perovskite oxide and transitionmetal dichalcogenides (TMDs) featured with unidirectional charge transfer enabling the in-situ local phase transition in TMDs. Differently, the LSC/K-MoSe ${ }_{2}$ system in this study characterizes the multidirectional charge transfer phenomenon, involving two-way charge transfer from $\mathrm{K}$ to $\mathrm{MoSe}_{2}$ and from LSC to $\mathrm{MoSe}_{2}$, which led to significantly improved water electrolysis performance and operational stability. When

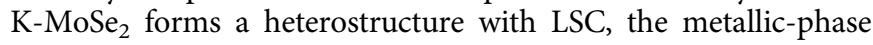
purity of $\mathrm{MoSe}_{2}$ is significantly increased to over $90 \%$ through the complementary charge transfer from LSC and potassium atoms. The optimized LSC/K-MoSe ${ }_{2}$ catalyst exhibits significantly enhanced HER and OER performance compared with those of LSC or $\mathrm{K}-\mathrm{MoSe}_{2}$, which is attributed to the increased electrical conductivity of $\mathrm{MoSe}_{2}$ and improved oxygen intermediate adsorption in LSC. In particular, the OER catalytic activity of $\mathrm{LSC} / \mathrm{K}-\mathrm{MoSe}_{2}$ outperforms that of the noble-metal $\mathrm{IrO}_{2}$ catalyst in $1 \mathrm{M} \mathrm{KOH}$. Consequently, the performance (e.g., overpotential and Tafel slope) and energy efficiency of overall water electrolysis of the LSC/K-MoSe ${ }_{2} \mid \mathrm{LSC} / \mathrm{K}-\mathrm{MoSe}_{2}$ couple surpass those of the state-of-the-art $\mathrm{Pt} / \mathrm{C} \| \mathrm{IrO}_{2}$ pair. Furthermore, the integrated overall water electrolysis exhibits excellent operational stability (over 2,500 h) without the decomposition of the catalyst under a high current density of $100 \mathrm{~mA} \mathrm{~cm}^{-2}$.

\section{Results}

Morphology and elemental characteristics of $\mathrm{LSC} / \mathrm{K}-\mathrm{MoSe}_{2}$. An $\mathrm{LSC} / \mathrm{K}-\mathrm{MoSe}_{2}$ heterostructure catalyst was synthesized via a simple and mass-producible solution process (Fig. 1a; see "Methods" section for details). In brief, LSC and K-MoSe $e_{2}$ were synthesized via the sol-gel method and molten-metal-assisted intercalation ${ }^{26}$, respectively; the as-prepared LSC and $\mathrm{K}-\mathrm{MoSe}_{2}$ were mixed via ball milling at specified weight percent ratios. The resulting $\mathrm{LSC} / \mathrm{K}-\mathrm{MoSe}_{2}$ compounds prepared in powder and solution forms are shown in Fig. 1a. Morphological and elemental analyses were conducted via scanning electron microscopy (SEM) and transmission electron microscopy (TEM). Supplementary Fig. 1 shows the SEM image of the LSC/K-MoSe 2 heterostructure, indicating that $\mathrm{K}-\mathrm{MoSe}_{2}$ was uniformly adsorbed onto the LSC surface without aggregation, which can contribute to the high specific surface area of the heterostructure. Figure $1 \mathrm{~b}$ shows the high-angle annular dark-field (HAADF) image of LSC/K-MoSe and the corresponding TEM energy-dispersive spectroscopy (EDS) elemental mapping profile. All relevant components (La, $\mathrm{Sr}, \mathrm{Co}, \mathrm{O}, \mathrm{Mo}$, Se, and $\mathrm{K}$ ) were uniformly distributed in the heterostructure, and the elemental compositions of each component of LSC/K-MoSe $e_{2}$ obtained from the TEM-EDS spectrum are presented in Supplementary Fig. 2 with the prescribed elemental ratio. High-resolution TEM (HR-TEM) analysis was further performed to observe the atomic structure of $\mathrm{LSC} / \mathrm{K}$ $\mathrm{MoSe}_{2}$ in detail. As shown in Fig. 1c-e, the coexistence of LSC and $\mathrm{K}-\mathrm{MoSe}_{2}$ can be clearly observed. LSC shows the well-known $\mathrm{ABO}_{3}$ layered perovskite oxide structure comprising $\mathrm{La}$ and $\mathrm{Sr}$ at the $\mathrm{A}$ site, $\mathrm{Co}$ at the $\mathrm{B}$ site, and $\mathrm{O}$ at the anion site, and the fast Fourier transform (FFT) pattern indicated a highly crystalline structure corresponding to the (110) and (001) planes of the LSC in the [110] zone axis (Fig. 1d). K-MoSe ${ }_{2}$ exhibits the typical 1Tphase atomic structure, and the FFT pattern on the [001] zone axis shows the desired crystalline structure of $\mathrm{K}-\mathrm{MoSe}_{2}$ corresponding to the (100) and (110) planes (Fig. 1e). The K-MoSe flakes formed on the as-synthesized $\mathrm{LSC} / \mathrm{K}-\mathrm{MoSe}_{2}$ are a few layers in thickness, indicating that the as-exfoliated $\mathrm{K}-\mathrm{MoSe}_{2}$ flakes were successfully integrated with the LSC without additional restacking (Supplementary Fig. 3).

Electrochemical performance. The electrochemical activity of $\mathrm{LSC} / \mathrm{K}-\mathrm{MoSe}_{2}$ was investigated by analyzing the linear sweep voltammetry (LSV) curve for HER and OER, cyclic voltammograms, double-layer capacitance values, and electrochemical impedance spectroscopy (EIS) results. As shown in Supplementary Figs. 4-7, Supplementary Tables 1 and 2, various weight percent ratios of LSC and K-MoSe 2 were first evaluated, and the 
optimal ratio of $\mathrm{LSC} / \mathrm{K}-\mathrm{MoSe}_{2}$ (5:4) yielding the best electrochemical catalytic performance was determined. This optimized configuration of $\mathrm{LSC} / \mathrm{K}-\mathrm{MoSe}_{2}$ heterostructure was utilized for further analyses. The hydrogen generation activity of LSC/K$\mathrm{MoSe}_{2}$ was evaluated in the half-cell system, and Fig. 2a compares the polarization curves of LSC/K-MoSe $2, \mathrm{~K}-\mathrm{MoSe}_{2}, \mathrm{LSC}$, and Pt/C in $\mathrm{N}_{2}$-saturated $1 \mathrm{M} \mathrm{KOH}$ using a three-electrode system. The corresponding Tafel slope of HER in various electrode configurations derived from the obtained LSV profile is shown in Fig. 2b. The Pt/C catalyst exhibited an overpotential of $68 \mathrm{mV}$ at

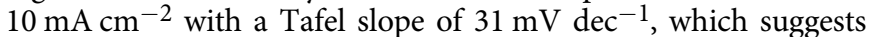
that the Volmer-Tafel reaction is a rate-determining step ${ }^{27}$. In the case of $\mathrm{LSC} / \mathrm{K}-\mathrm{MoSe}_{2}$, the catalyst exhibited significantly higher HER activity than that of the LSC and K-MoSe 2 . LSC/K$\mathrm{MoSe}_{2}$ showed an overpotential of $128 \mathrm{mV}$ at $10 \mathrm{~mA} \mathrm{~cm}^{-2}$ and a Tafel slope of $45 \mathrm{mV} \mathrm{dec}-1$, whereas $\mathrm{K}-\mathrm{MoSe}_{2}$ and LSC required 288 and $450 \mathrm{mV}$ to reach $10 \mathrm{~mA} \mathrm{~cm}^{-2}$ with Tafel slopes of 62 and $119 \mathrm{mV} \mathrm{dec}^{-1}$, respectively. The considerably improved Tafel slope, and thus the HER performance, of LSC/K-MoSe ${ }_{2}$ implies that the primary rate-determining step of the heterostructured catalyst becomes closer to the Volmer-Tafel pathway of noble metals ${ }^{28,29}$. The enhanced HER performance of $\mathrm{LSC} / \mathrm{K}-\mathrm{MoSe}_{2}$ was also confirmed through charge transfer resistance $\left(\mathrm{R}_{\mathrm{ct}}\right)$ analysis between the electrode and electrolyte, which can directly affect electrochemical performance. Figure $2 c$ shows the Nyquist plots of LSC/K-MoSe $2, \mathrm{~K}-\mathrm{MoSe}_{2}$, and LSC for the HER obtained via EIS analysis, and the derived $R_{\mathrm{ct}}$ values were $1.30,2.56$, and $5.78 \Omega \mathrm{cm}^{2}$, respectively, which corroborates that charge transfer kinetics is most favorable for $\mathrm{LSC} / \mathrm{K}-\mathrm{MoSe}_{2}$ than others. We also investigated the OER activity of various catalyst configurations in $\mathrm{N}_{2}$-saturated $1 \mathrm{M} \mathrm{KOH}$ using a three-electrode system. Figure $2 \mathrm{~d}$ shows the LSV curves of LSC/K-MoSe $, \mathrm{K}_{2}-\mathrm{MoSe}_{2}, \mathrm{LSC}$, and $\mathrm{IrO}_{2}$ for OER. The corresponding Tafel slope is shown in Fig. 2e. $\mathrm{IrO}_{2}$, known as the best performing noble-metal-based catalyst in $\mathrm{OER}^{5}$, showed an overpotential of $350 \mathrm{mV}$ at $10 \mathrm{~mA} \mathrm{~cm}^{-2}$ with a Tafel slope of $81 \mathrm{mV} \mathrm{dec}^{-1}$. Notably, the OER catalytic performance of $\mathrm{LSC} / \mathrm{K}-\mathrm{MoSe}_{2}$ exceeded that of $\mathrm{IrO}_{2}$ with an overpotential as low as $230 \mathrm{mV}$ to reach $10 \mathrm{~mA} \mathrm{~cm}^{-2}$ and a Tafel slope of $79 \mathrm{mV} \mathrm{dec}^{-1}$. In contrast, LSC required an overpotential of $420 \mathrm{mV}$ to reach $10 \mathrm{~mA} \mathrm{~cm}^{-2}$ with a Tafel slope of $131 \mathrm{mV}$ $\mathrm{dec}^{-1}$ and $\mathrm{K}-\mathrm{MoSe}_{2}$ exhibited negligible OER activity. It has been reported that the OER performance of perovskite oxide can be improved by the doping effect of molybdenum ${ }^{30}$. We thus conducted XPS analysis of Mo and Se in LSC/K-MoSe $e_{2}$ under OER condition after water electrolysis to elucidate the potential doping effect of Mo on the OER performance of LSC/K-MoSe $e_{2}$ (Supplementary Fig. 8 and Supplementary Table 3). The XPS results indicated partial oxidation of Mo and Se, but not the decomposition, in $\mathrm{LSC} / \mathrm{K}-\mathrm{MoSe}_{2}$ in our proposed heterostructured electrocatalyst system. In general, molybdenum oxide is known to have negligible OER activity in an alkaline environment, which is consistent with the observation of poor OER performance of $\mathrm{K}-\mathrm{MoSe}_{2}$ in our study ${ }^{31}$. Thus, the origin of OER performance enhancement of LSC in the heterostructure is not attributed to the doping effect of molybdenum or its oxide derivatives. In our system, Mo, rather than serving as the main contributor for the OER active site, is believed to participate in the continuous charge transfer process with LSC during the OER reaction, which enhances the electrophilicity of LSC, leading to the increased adsorption of OER intermediates such as $\mathrm{OH}^{*}$ and $\mathrm{OOH}^{*}$, and modulates the electronic structure of LSC/K-MoSe ${ }_{2}$ favorable for OER process. The improvement in OER kinetics of $\mathrm{LSC} / \mathrm{K}-\mathrm{MoSe}_{2}$ compared to that of LSC owing to the presence of Mo in the OER environment was also previously verified via experimental and computational analyses (low Tafel slope value (Fig. 2e), upshift of XPS spectra of Co 2p peak (Supplementary Fig. 14), and reduced free energy barrier and density of states (Fig. $4 \mathrm{f}-\mathrm{h}$ and Supplementary Figs. 21-23)). The $R_{\mathrm{ct}}$ values of $\mathrm{LSC} / \mathrm{K}-\mathrm{MoSe}_{2}, \mathrm{~K}-\mathrm{MoSe}_{2}$, and LSC for OER obtained from the EIS analysis were 1.52, 74.0, and $1.91 \Omega \mathrm{cm}^{2}$, respectively (Fig. 2f). The lower $R_{\mathrm{ct}}$ in LSC/K$\mathrm{MoSe}_{2}$ than $\mathrm{K}-\mathrm{MoSe}_{2}$ and LSC indicates that the interfacial resistance between the electrode and electrolyte is reduced through heterostructure formation, contributing to the improved OER kinetics. These results suggest that the formation of the LSC/ $\mathrm{K}-\mathrm{MoSe}_{2}$ heterostructure may induce potential physicochemical interactions between the LSC and $\mathrm{K}-\mathrm{MoSe}_{2}$, generating synergistic effects to lead to the observed excellent intrinsic catalytic activities for both the HER and OER processes. Below, we present detailed analyses on the heterogeneous composite structure to elucidate the origin of the enhanced catalytic performance.

Analysis of physical properties of $\mathbf{L S C} / \mathrm{K}-\mathrm{MoSe}_{2}$. The crystal structures of $2 \mathrm{H}-\mathrm{MoSe}_{2}, \mathrm{LSC} / \mathrm{K}-\mathrm{MoSe}_{2}, \mathrm{~K}-\mathrm{MoSe}_{2}$, and LSC were investigated via X-ray diffraction (XRD). As shown in Fig. 3a, the preferred crystallographic orientation of $2 \mathrm{H}-\mathrm{MoSe}_{2}$ at (002) plane is observed at $13.8^{\circ 32}$, whereas that of $\mathrm{K}-\mathrm{MoSe}_{2}$ is blue shifted to $13.4^{\circ}$ owing to the increased interlayer spacing between the $\mathrm{MoSe}_{2}$ layers through the formation of potassium-intercalated $\mathrm{MoSe}_{2}$. The preferred orientation of the (002) plane for $\mathrm{K}-\mathrm{MoSe}_{2}$ was consistently found at $13.4^{\circ}$ for the $\mathrm{LSC} / \mathrm{K}-\mathrm{MoSe}_{2}$ heterostructure. In addition, as shown in Supplementary Fig. 9, the crystal structures of $\mathrm{K}-\mathrm{MoSe}_{2}$ and LSC can be clearly identified from $\mathrm{LSC} / \mathrm{K}-\mathrm{MoSe}_{2}$, indicating that each crystal structure of $\mathrm{K}$ $\mathrm{MoSe}_{2}$ and LSC is well preserved when forming the heterostructure through the ball mill process. Thermogravimetric analysis (TGA) was performed to examine the surface adsorption capability of the catalysts (Fig. 3b). Target materials, including $\mathrm{LSC} / \mathrm{K}-\mathrm{MoSe}_{2}, \mathrm{~K}-\mathrm{MoSe}_{2}$, and LSC, were preexposed to wet-air conditions to adsorb various environmental species in the atmosphere such as moisture, $\mathrm{H}$, and $\mathrm{OH}$ groups. With incremental heat treatments up to $600{ }^{\circ} \mathrm{C}$, LSC/K-MoSe $e_{2}$ exhibited a weight loss of $\sim 14 \%$, whereas both $\mathrm{K}-\mathrm{MoSe}_{2}$ and LSC exhibited only a marginal weight loss of $\sim 2 \%$, demonstrating the improved surface adsorption capability of the heterostructure. Brunauer-Emmett-Teller (BET) analysis was conducted to investigate the specific surface area of $\mathrm{LSC} / \mathrm{K}-\mathrm{MoSe}_{2}$ (Fig. 3c). Compared with $\mathrm{K}-\mathrm{MoSe}_{2}$ and LSC, the heterostructure exhibited significantly enhanced surface areas, i.e., 203.94, 104.51, and $32.54 \mathrm{~m}^{2} \mathrm{~g}^{-1}$ for LSC/K-MoSe $2, \mathrm{~K}-\mathrm{MoSe}_{2}$, and LSC, respectively, indicating that the active sites of the heterostructured catalyst can be increased to improve the efficiency of the electrochemical cell. We further analyzed the BET surface area and water electrolysis performance of various other conditioned samples comprising LSC and $\mathrm{K}-\mathrm{MoSe}_{2}$ to gain more insights for the surface area effect according to different ball-milling processes on the water electrolysis performance (Supplementary Figs. 10, 11 and Supplementary Table 4). We note that, overall, there was no significant difference in the morphology for each conditioned samples before and after the ball-milling process (Supplementary Fig. 12). For the individual component materials (LSC and $\mathrm{MoSe}_{2}$ ), the ballmilled samples showed slightly increased BET surface areas, but the water electrolysis performance remained almost unaffected. When forming the heterostructured catalyst, the ball-milling process to the specific surface area and the electrolytic performance exhibited the most synergistic effects when applied during the heterostructure formation of individual component materials (i.e., LSC and $\mathrm{K}-\mathrm{MoSe}_{2}$ ) rather than to each component of LSC and $\mathrm{K}-\mathrm{MoSe}_{2}$ followed with the mixing process. These results indicate that ball milling during the formation of the composite structures increases the active sites for the water electrolysis and facilitates the charge transfer between LSC and $\mathrm{K}-\mathrm{MoSe}_{2}$, thereby 
a
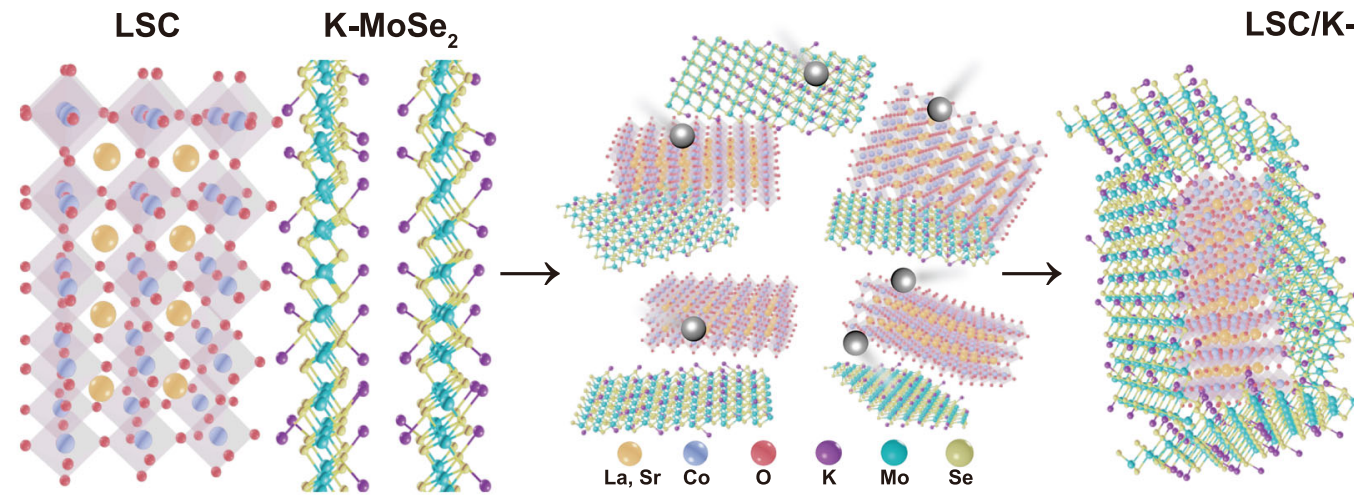

$\mathrm{LSC} / \mathrm{K}-\mathrm{MoSe}_{2}$

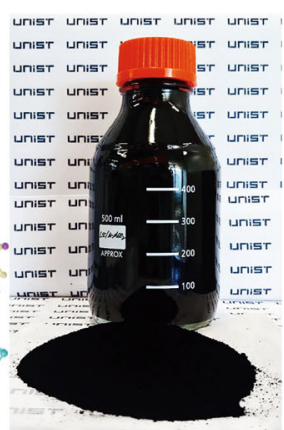

b
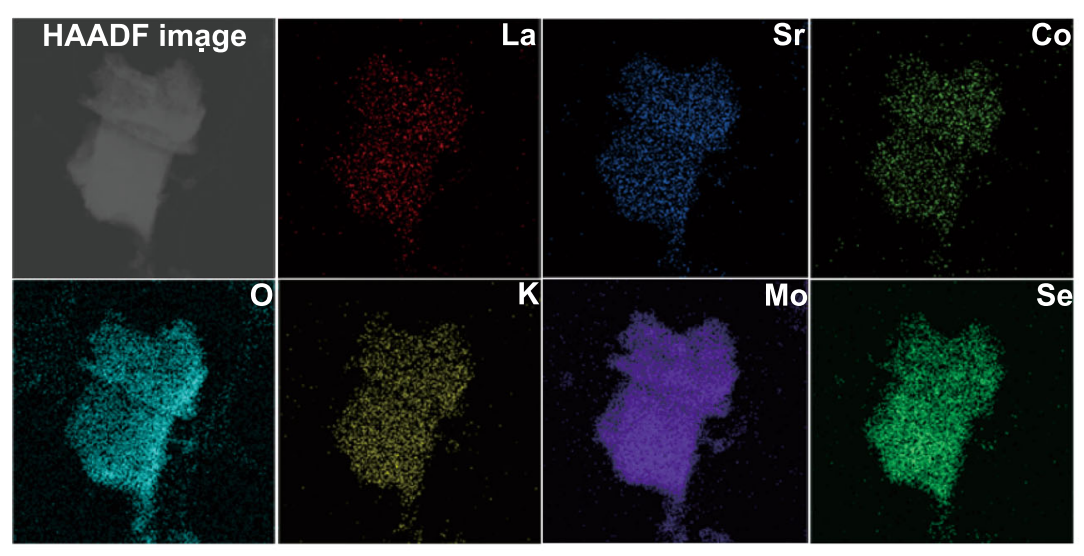

C

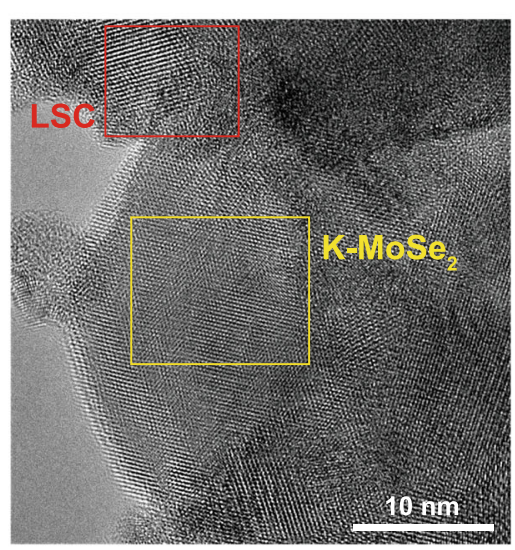

d

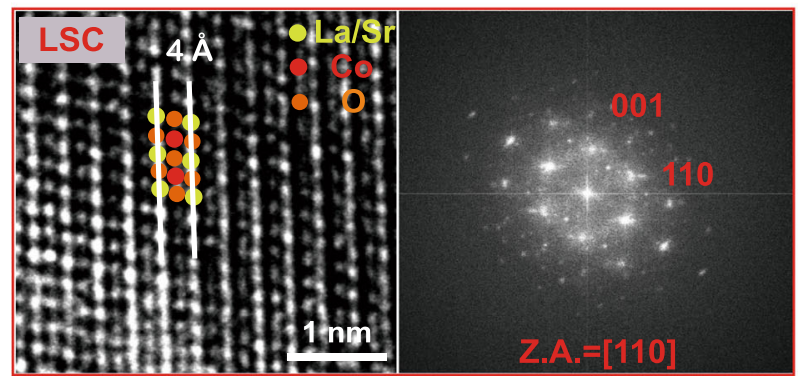

e

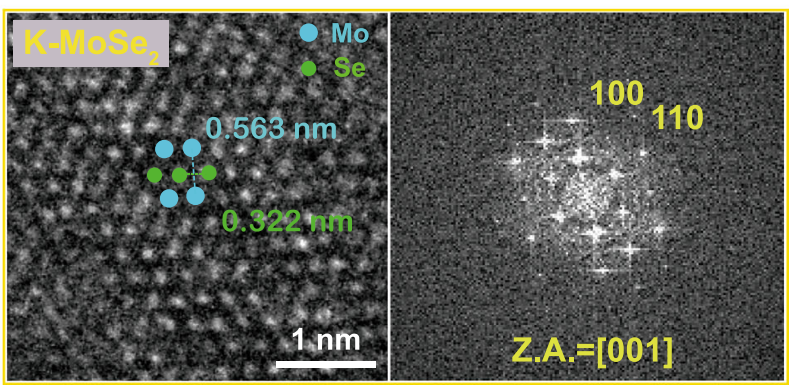

Fig. 1 Elemental and morphological characterizations of $\mathbf{L S C} / \mathbf{K}-\mathbf{M o S e}_{\mathbf{2}}$ heterostructure. a Schematic illustrating the synthesis process of LSC/K-MoSe ${ }_{2}$. Digital image demonstrates the large-scale synthesis capability of the proposed method. b HAADF-STEM image and corresponding EDS elemental mapping of La (red), Sr (blue), Co (yellow green), O (cyan), K (yellow), Mo (purple), and Se (green). c HR-TEM image of LSC/K-MoSe 2 , showing typical morphological characteristics of the heterostructure comprising the LSC (red square) and $\mathrm{K}-\mathrm{MoSe}_{2}$ (yellow square) regions. d Representative HR-TEM image of LSC and corresponding FFT image, indicating the lattice structure of La/Sr, Co, and O with high crystallinity. e Representative HR-TEM image of $\mathrm{K}-\mathrm{MoSe}_{2}$ and corresponding FFT image, exhibiting the highly crystalline lattice structure of $1 \mathrm{~T}$-phase $\mathrm{MoSe}_{2}$ comprising Mo and Se.

enabling the improved water electrolysis performance in the heterostructured catalyst. In addition, the pore size of LSC/K$\mathrm{MoSe}_{2}$ was measured using the Barrett-Joyner-Halenda (BJH) method. LSC/K-MoSe $e_{2}$ exhibited a mesoporous pore size distribution of $2-50 \mathrm{~nm}$ (Supplementary Fig. 13). The mesoporous nature of the catalyst can effectively improve the surface area of the catalyst as well as the diffusion of the electrolyte and ionic species $^{33}$. Further, the desorption of hydrogen and oxygen generated during the overall water electrolysis reaction becomes favorable owing to the rapid mass transfer from the electrolyte to the catalyst surface, which can help to improve the performance of the water electrolysis ${ }^{34,35}$.

Figure 3d shows the Raman spectra of $\mathrm{LSC} / \mathrm{K}-\mathrm{MoSe}_{2}$ and $\mathrm{K}-\mathrm{MoSe}_{2}$. Characteristic vibrational Raman modes of $\mathrm{K}-\mathrm{MoSe}_{2}$ were detected at 106.2, 150.5, 221.6, 165.2 , and $289.7 \mathrm{~cm}^{-1}$, corresponding to the $\mathrm{J}_{1}, \mathrm{~J}_{2}, \mathrm{~J}_{3}, \mathrm{E}_{1 \mathrm{~g}}$, and $\mathrm{E}_{2 \mathrm{~g}}{ }_{2 \mathrm{~g}}$ peaks of $1 \mathrm{~T}-\mathrm{MoSe}_{2}{ }^{36}$. The characteristic
Raman peak positions of $\mathrm{LSC} / \mathrm{K}-\mathrm{MoSe}_{2}$ were red shifted by $2 \mathrm{~cm}^{-1}$ indicating potential electronic interaction between the LSC and K$\mathrm{MoSe}_{2}{ }^{37}$. Ultraviolet photoelectron spectroscopy (UPS) analysis was performed to investigate the charge transfer effect between LSC and $\mathrm{K}-\mathrm{MoSe}_{2}$. Work function values were derived from the secondary cutoff energies. Figure $3 e$ shows that the work function of $\mathrm{LSC} / \mathrm{K}$ $\mathrm{MoSe}_{2}$ increased compared to LSC and K-MoSe $e_{2}$ alone (5.38 eV vs. 5.15 and $5.22 \mathrm{eV}$ ). This verifies that electronic structure modulation in LSC/K-MoSe $e_{2}$ occurs via charge transfer between LSC and K$\mathrm{MoSe}_{2}$. Compared with LSC, LSC/K-MoSe $e_{2}$ with an increased work function has several merits for improving electrochemical performance. The increased work function enhances the rate constant and preexponential kinetic factor in the electrocatalytic reaction ${ }^{38,39}$. The high rate constant and kinetic factor reduce the bond strength between active sites and adsorbed intermediates of HER on the catalyst surface, increasing the exchange current density and enabling 
a

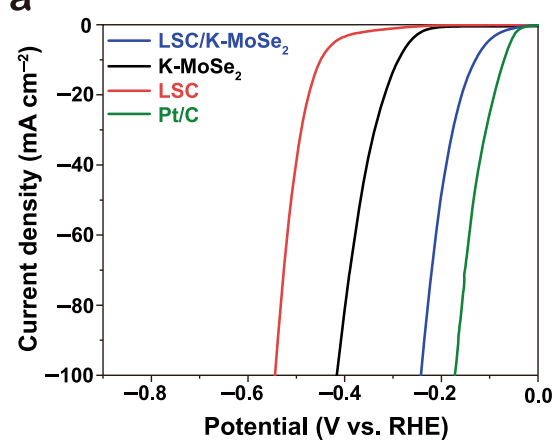

d

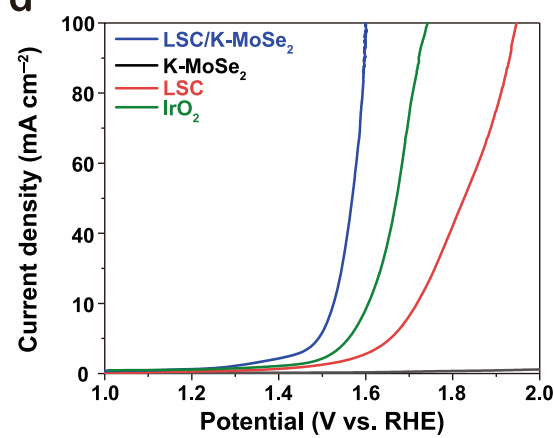

b

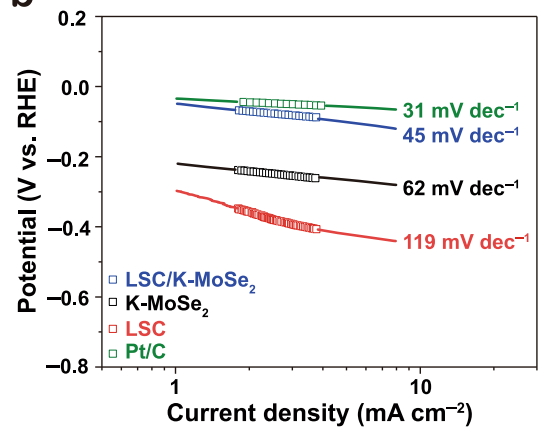

e

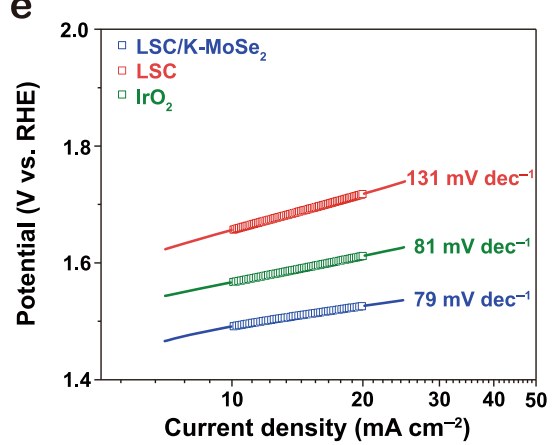

C

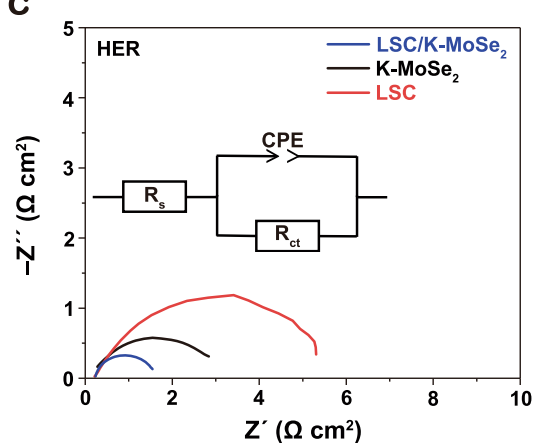

f

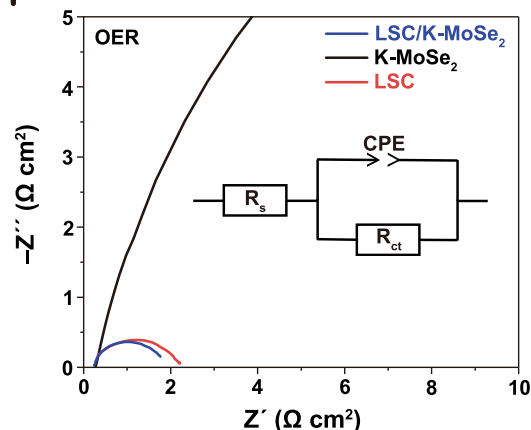

Fig. 2 Electrocatalytic HER and OER performances of $\mathbf{L S C} / \mathbf{K}-\mathbf{M o S e}_{\mathbf{2}}, \mathbf{K}-\mathbf{M o S e}_{\mathbf{2}}$, and LSC. a, d HER and OER polarization curves recorded in $\mathrm{N}_{2}$-saturated 1 $\mathrm{M} \mathrm{KOH}$ at a scan rate of $5 \mathrm{mV} \mathrm{s}^{-1}$. b, e Corresponding Tafel slopes of the HER and OER profiles derived from the polarization curves. $\mathbf{c}, \mathbf{f}$ EIS analysis of HER and OER for LSC/K-MoSe, $\mathrm{K}-\mathrm{MoSe}_{2}$, and LSC. The Nyquist plots comprise real ( $\left.Z^{\prime}\right)$ and imaginary ( $\left.Z^{\prime \prime}\right)$ parts fitted as $x$ and $y$ axes, respectively.

the Gibbs free energy of hydrogen adsorbed on the active site closer to the thermoneutral point $(\sim 0 \mathrm{eV})$. It also enables overpotential reduction for the Volmer reaction owing to the increase in proton concentration of the electronic double layer of the catalyst, thereby improving HER performance ${ }^{40}$. UV-Vis-NIR spectroscopy analysis in Fig. 3f reveals that the $\mathrm{A}$ and $\mathrm{B}$ excitonic peaks observed for semiconducting $2 \mathrm{H}-\mathrm{MoSe}_{2}{ }^{41}$ disappear in $\mathrm{LSC} / \mathrm{K}-\mathrm{MoSe}_{2}$ and $\mathrm{K}$ $\mathrm{MoSe}_{2}$, illustrating the metal-like characteristics of the as-synthesized $\mathrm{LSC} / \mathrm{K}-\mathrm{MoSe}_{2}$ and $\mathrm{K}-\mathrm{MoSe}_{2}{ }^{42}$.

$\mathrm{X}$-ray photoelectron spectroscopy (XPS) analysis was performed to further elucidate the origin of the enhanced HER and OER performance of $\mathrm{LSC} / \mathrm{K}-\mathrm{MoSe}_{2}$. First, we analyzed the chemical states of Co $2 p$ and O $1 s$ core levels of LSC and LSC/ $\mathrm{K}-\mathrm{MoSe}_{2}$ to infer the cause of catalytic electrolysis performance improvement. The Co $2 \mathrm{p}$ peaks of LSC and $\mathrm{LSC} / \mathrm{K}-\mathrm{MoSe}_{2}$ include Co $2 p^{3+}$, Co $2 p^{2+}$, and two satellite features. As shown in Supplementary Fig. 14, in LSC, $\mathrm{Co}^{3+}$ and $\mathrm{Co}^{2+}$ peaks are located at 779.6/794.2 and 781.5/797.0 eV, and those of $\mathrm{LSC} / \mathrm{K}-\mathrm{MoSe}_{2}$ are located at 780.6/795.2 and 782.5/798.0 eV, respectively, illustrating an upshift of $1 \mathrm{eV}$ in the peak position for the heterostructure. This upshift can be attributed to the electronic interaction between LSC and $\mathrm{K}-\mathrm{MoSe}_{2}$. In $\mathrm{LSC} / \mathrm{K}-\mathrm{MoSe}_{2}$, a difference in the electronegativity between Mo and Co induces charge transfer, which can modulate the electronic structure ( $e_{g}$ orbital) of Co while maintaining the overall electroneutrality. As the $\mathrm{e}_{\mathrm{g}}$-orbital filling of perovskite oxide affects the binding of oxygen-related intermediates at the active site (typically at B site), optimizing the $e_{\mathrm{g}}$-orbital occupancy close to 1 is critical for achieving optimal OER performance ${ }^{43}$. In Co, which is the B site of LSC, $\mathrm{e}_{\mathrm{g}}$-orbital fillings of $\mathrm{Co}^{3+}\left(t^{5}{ }_{2 g} e^{1}\right)$ and $\mathrm{Co}^{2+}\left(t^{5}{ }_{2 g} e^{2}{ }_{g}\right)$ are 1 and 2 , respectively, which suggests that increasing the $\mathrm{Co}^{3+}$ proportion over $\mathrm{Co}^{2+}$ is preferable to obtain the optimized $\mathrm{e}_{\mathrm{g}}$-orbital occupancy ${ }^{44,45}$. The ratio of $\mathrm{Co}^{3+} / \mathrm{Co}^{2+}$, the value obtained from the XPS spectra, is summarized in Supplementary Table 5; this ratio was 1.5 and 2.4 for LSC and LSC/K-MoSe 2 , respectively.
Therefore, near-unity $\mathrm{e}_{\mathrm{g}}$-orbital occupancy can be expected for LSC/K-MoSe $e_{2}$, confirming improved OER performance in the heterostructure. The chemical state of $\mathrm{O} 1 \mathrm{~s}$ in the catalyst can also directly affect OER kinetics ${ }^{46,47}$. As shown in Supplementary Fig. 15, O $1 s$ peaks of LSC and LSC/K-MoSe ${ }_{2}$ include four secondary (shoulder) peaks comprising the lattice oxygen at $528.31 \mathrm{eV}\left(\mathrm{O}_{2}{ }^{-}\right.$, denoted as $\left.\mathrm{LO}\right)$, highly oxidative oxygen species at $531.1 \mathrm{eV}\left(\mathrm{O}_{2}{ }^{2-} / \mathrm{O}^{-}\right.$, denoted as $\left.\mathrm{OO}\right)$, surface adsorbed oxygen including hydroxyl groups at $532.11 \mathrm{eV}\left(\mathrm{O}_{2} / \mathrm{OH}^{-}\right.$, denoted as $\mathrm{SO})$, and adsorbed molecular water at $533.2 \mathrm{eV}\left(\mathrm{H}_{2} \mathrm{O}\right.$, denoted as AW). The larger amount of surface adsorbed oxygen species compared to the lattice oxygen on the catalyst surface has a favorable effect on the formation of oxygen vacancy and ratedetermining step of the OER process ${ }^{46,47}$. Thus, the increased SO/ $\mathrm{LO}$ ratio of $\mathrm{LSC} / \mathrm{K}-\mathrm{MoSe}_{2}$ over LSC indicates that the formation kinetics of the active sites- $\mathrm{O},-\mathrm{OH}$, and $-\mathrm{OO}$ bonds for the heterostructure was improved, which can contribute to the enhanced electrolytic performance in alkaline solutions (Supplementary Table 6).

The chemical state of $\mathrm{MoSe}_{2}$ was also investigated to further elucidate the origin of electrochemical performance improvement in the heterostructure. As shown in Fig. 3g, h, 1T- and 2H-phase $\mathrm{MoSe}_{2}$ with different relative ratios coexist in LSC/K-MoSe ${ }_{2}$ and $\mathrm{K}-\mathrm{MoSe}_{2}$. The XPS peaks of the 1T-phase in these configurations are positioned at 228.3 and $231.4 \mathrm{eV}$ for Mo $3 d_{5 / 2}$ and Mo $3 d_{3 / 2}$, and at 53.7 and $54.7 \mathrm{eV}$ for Se $3 d_{5 / 2}$ and Se $3 d_{3 / 2}$, whereas those of the $2 \mathrm{H}$-phase are located at 229.0 and $232.8 \mathrm{eV}$ for Mo $3 d_{5 / 2}$ and Mo $3 d_{3 / 2}$, and at 54.3 and $55.8 \mathrm{eV}$ for Se $3 d_{5 / 2}$ and Se $3 d_{3 / 2}$, respectively. Figure $3 \mathrm{i}$ summarizes the relative contents of the $1 \mathrm{~T}$ and $2 \mathrm{H}$-phase $\mathrm{MoSe}_{2}$ for as-prepared $\mathrm{LSC} / \mathrm{K}-\mathrm{MoSe}_{2}$ and $\mathrm{K}$ $\mathrm{MoSe}_{2}$ obtained from the XPS spectra. The high-purity 1T-phase $\mathrm{MoSe}_{2}(\sim 91 \%)$ found in the heterostructure over that of $\mathrm{K}-\mathrm{MoSe}_{2}$ $(\sim 67 \%)$ clearly evidence that electronic interaction occurs between LSC and $\mathrm{K}-\mathrm{MoSe}_{2}$ causing the further metallic-phase transition of $\mathrm{MoSe}_{2}$ in $\mathrm{K}-\mathrm{MoSe}_{2}$. 


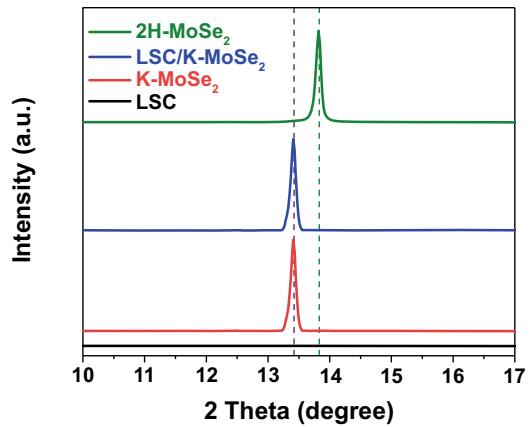

d

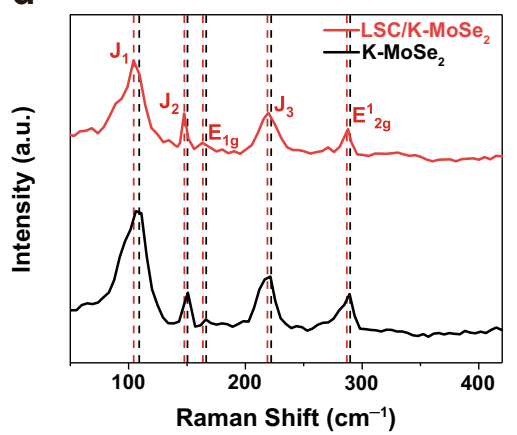

g

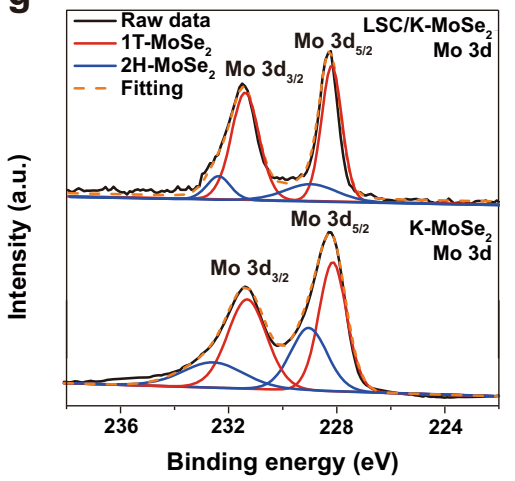

b

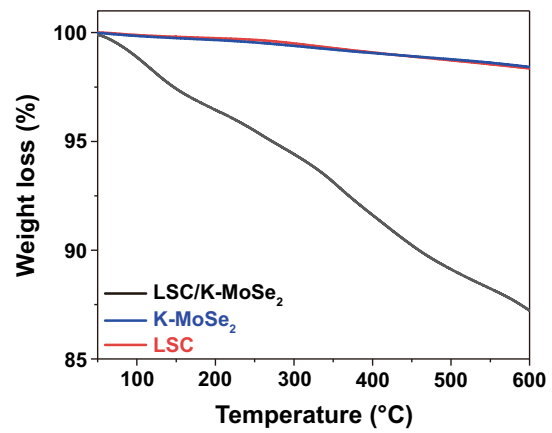

e

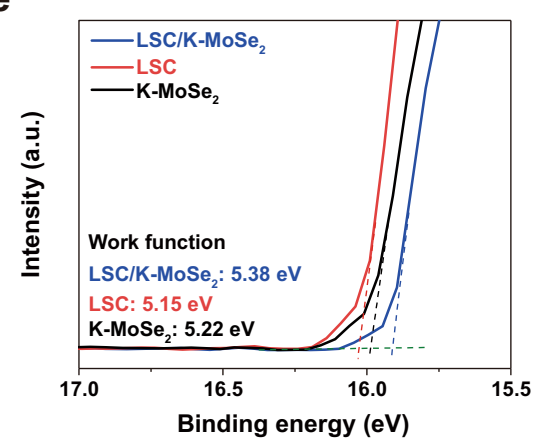

h

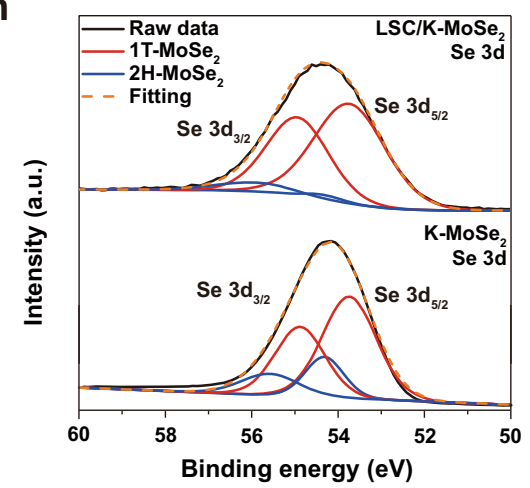

C

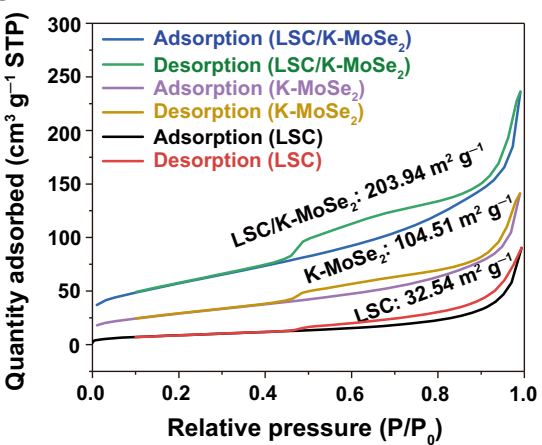

f

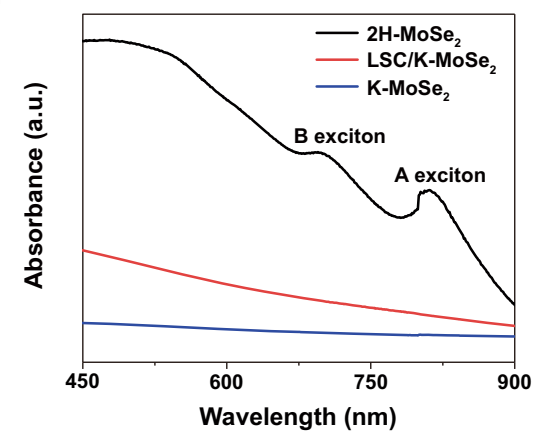

i

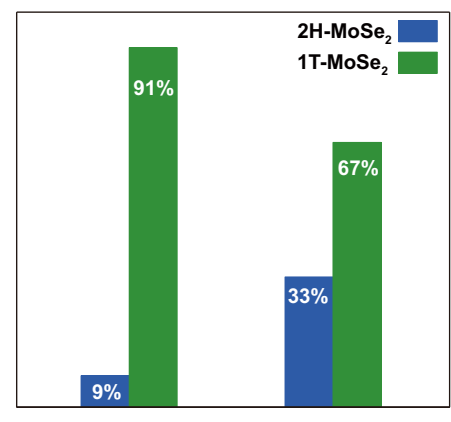

LSC/K-MoSe

K-MoSe

Fig. 3 Characterization of the $\mathbf{L S C} / \mathbf{K}-\mathbf{M o S e}_{\mathbf{2}}$ heterostructure. a XRD patterns for $2 \mathrm{H}-\mathrm{MoSe}_{2}, \mathrm{LSC} / \mathrm{K}-\mathrm{MoSe}_{2}, \mathrm{~K}-\mathrm{MoSe}_{2}$, and LSC, demonstrating that each of the $\mathrm{K}-\mathrm{MoSe}_{2}$ and LSC phases are well preserved in LSC/K-MoSe 2 . b TGA analysis of LSC/K-MoSe $e_{2}, \mathrm{~K}-\mathrm{MoSe}_{2}$, and LSC, indicating the improved surface adsorption capability of $\mathrm{LSC} / \mathrm{K}-\mathrm{MoSe}_{2}$. c BET surface area of $\mathrm{LSC} / \mathrm{K}-\mathrm{MoSe}_{2}, \mathrm{~K}-\mathrm{MoSe}_{2}$, and $\mathrm{LSC}$ obtained from $\mathrm{N}_{2}$ adsorption/desorption isotherms. d Raman spectra of $\mathrm{LSC} / \mathrm{K}-\mathrm{MoSe}_{2}$ and $\mathrm{K}-\mathrm{MoSe}_{2}$, illustrating the electronic interaction between $\mathrm{LSC}$ and K-MoSe${ }_{2}$. e UPS valence band spectra of LSC/K$\mathrm{MoSe}_{2}, \mathrm{LSC}$, and K-MoSe . f UV-Vis-NIR spectra of $2 \mathrm{H}-\mathrm{MoSe}_{2}, \mathrm{LSC} / \mathrm{K}-\mathrm{MoSe}_{2}$, and K-MoSe $e_{2}$ indicating the metallic features of LSC/K-MoSe 2 and K$\mathrm{MoSe}_{2} . \mathbf{g}, \mathbf{h}$ High-resolution XPS spectra of Mo $3 \mathrm{~d}$ and Se 3d peaks for LSC/K-MoSe $e_{2}$ and K-MoSe 2 . i Relative fraction of $2 \mathrm{H}-$ and $1 \mathrm{~T}-\mathrm{MoSe} \mathrm{H}_{2}$ in LSC/K$\mathrm{MoSe}_{2}$ and $\mathrm{K}-\mathrm{MoSe}_{2}$, indicating the substantial increase of the $1 \mathrm{~T}$-phase ratio in $\mathrm{MoSe}_{2}$ through complementary charge transfer.

Complementary charge transfer in $\mathrm{LSC} / \mathrm{K}-\mathrm{MoSe}_{2}$. We hypothesize that the drastic increase in the 1T-phase content of $\mathrm{MoSe}_{2}$ in the heterostructured catalyst occurs because of the complementary charge transfer between LSC and $\mathrm{K}-\mathrm{MoSe}_{2}$, as schematically illustrated in Fig. 4a. K-MoSe $e_{2}$ is synthesized by intercalating the potassium metal within the $\mathrm{MoSe}_{2}$ interlayers, followed by subsequent exfoliation, where potassium atoms and Se form K-Se ionic bonds. The large electronegativity difference (1.73) between $\mathrm{K}$ and Se promotes the charge transfer from $\mathrm{K}$ to $\mathrm{Se}$, modulating the Mo $4 d$ orbital configuration of $\mathrm{MoSe}_{2}$ from the occupied $4 d_{z}{ }^{2}$ level to incompletely filled $4 d_{x z}, 4 d_{y z}$, and $4 d_{y x}$ orbitals. This electronic structure rearrangement causes local phase transition from $2 \mathrm{H}-\mathrm{MoSe}_{2}$ to $1 \mathrm{~T}-\mathrm{MoSe}_{2}$. Following the formation of the LSC/K-MoSe $e_{2}$ heterostructure, charge transfer from the Co of LSC to the $2 \mathrm{H}-\mathrm{MoSe}_{2}$ portion of $\mathrm{K}-\mathrm{MoSe}_{2}$ occurs, causing an additional 1T-phase transition in $\mathrm{MoSe}_{2}$. This complementary charge transfer in LSC/K-MoSe 2 has beneficial effects on both HER and OER performance. The increased 1Tphase $\mathrm{MoSe}_{2}$ concentration in $\mathrm{LSC} / \mathrm{K}-\mathrm{MoSe}_{2}$ creates a more electron-rich and electrically conducting surface, enabling fast electron transfer at the catalytically active site, thereby improving HER kinetics ${ }^{48}$. The Co in LSC, after donating the electrons to K$\mathrm{MoSe}_{2}$, becomes more electrophilic; such charge transfer results in the upshift of the $d$-band center and improved adsorption capability of oxygen-generating intermediates (e.g., $\mathrm{O}^{*}, \mathrm{OH}^{*}$, $\mathrm{OO}^{*}$ ) at the catalyst surface ${ }^{49}$, thereby enhancing OER kinetics.

We computationally analyzed charge transfer and catalytic activities in LSC/K-MoSe 2 using density functional theory (DFT) calculations. The evaluated charge transfer into a $\mathrm{MoSe}_{2}$ monolayer from Bader charge analysis ${ }^{50}$ is $2.48 e(0.88 e$ and $1.60 e$ from the $\mathrm{K}$ atom and LSC perovskite, respectively) in the $\mathrm{LSC} / \mathrm{K}-\mathrm{MoSe}_{2}$ structure model (Fig. 4b). We found that the 
charge transfer from LSC to $\mathrm{MoSe}_{2}$ is unaffected by the $\mathrm{K}$ atoms because the LSC to $\mathrm{MoSe}_{2}$ charge transfer in the $\mathrm{LSC} / \mathrm{MoSe}_{2}$ structure is the same value of $1.60 e$, indicating that the complementary charge transfer from K and LSC into $\mathrm{MoSe}_{2}$ is available in the $\mathrm{LSC} / \mathrm{K}-\mathrm{MoSe}_{2}$ system. The additional electron transfer from $\mathrm{K}$ into $\mathrm{MoSe}_{2}$ layers can lead to a larger portion of $1 \mathrm{~T}-\mathrm{MoSe}_{2}$ in LSC/K-MoSe $e_{2}$ than in LSC/MoSe $e_{2}$, supporting the experimentally observed results (Fig. $3 \mathrm{i}$ ). We found that the charge transfer from LSC to $\mathrm{MoSe}_{2}$ mainly occurs in the $\mathrm{CoO}_{2}$ subsurface layer of LSC as depicted in Fig. 4c, wherein drastic changes in the electron density are observed in the $\mathrm{CoO}_{2}$ layer; these changes are expected to boost OER performance with a change in the electronic structure of LSC layers. Charge transfer from the $\mathrm{CoO}_{2}$ layer to $\mathrm{MoSe}_{2}$ was also confirmed via the density of states (DOS) analysis presented in Supplementary Fig. 16 and Supplementary Note 2. The $\mathrm{CoO}_{2}$ layer under the outermost $\mathrm{La}_{0.5} \mathrm{Sr}_{0.5} \mathrm{O}$ surface plays an important role in charge transfer while electrocatalytic activities occur on the $\mathrm{La}_{0.5} \mathrm{Sr}_{0.5} \mathrm{O}$ surface, which is essential in explaining the superiority of LSC perovskite. Furthermore, we found that the complementary charge transfer from K and LSC into $\mathrm{MoSe}_{2}$ layers markedly reduced the energy barrier when $\mathrm{MoSe}_{2}$ underwent $2 \mathrm{H}$ - to $1 \mathrm{~T}$-phase transition, as shown in Fig. $4 \mathrm{~d}\left(0.65 \mathrm{eV}\right.$ in $\mathrm{MoSe}_{2}$ to $0.18 \mathrm{eV}$ in $\left.\mathrm{LSC} / \mathrm{K}-\mathrm{MoSe}_{2}\right)$; this indicates that a high portion of $1 \mathrm{~T}-\mathrm{MoSe}_{2}$ phase will be present in the $\mathrm{LSC} / \mathrm{K}-\mathrm{MoSe}_{2}$ heterostructure.

To further elucidate the enhanced water-splitting performance in $\mathrm{LSC} / \mathrm{K}-\mathrm{MoSe}_{2}$ heterostructure, we analyzed the reaction-free energy for subreactions in both HER and OER. The values of $\Delta G_{\mathrm{H}^{*}}$ can directly evaluate HER performance ${ }^{51-53}$. Figure $4 \mathrm{e}$ shows a comparison of $\Delta G_{\mathrm{H}^{*}}$ in LSC/K-MoSe ${ }_{2}$ with LSC, $2 \mathrm{H}-$ $\mathrm{MoSe}_{2}$, and $\mathrm{K}-\mathrm{MoSe}_{2}$. The LSC/K-MoSe 2 structures are suitable catalysts for providing the most favorable HER environment for $\mathrm{H}_{2}$ production with the smallest $\Delta G_{\mathrm{H}^{*}}$ of $0.25 \mathrm{eV}$, whereas the $\Delta G_{\mathrm{H}^{*}}$ values for LSC, $2 \mathrm{H}-\mathrm{MoSe}_{2}$, and K-MoSe $\mathrm{e}_{2}$ are 2.01, 1.44, and $0.69 \mathrm{eV}$, respectively. The optimal near-zero $\Delta G_{\mathrm{H}^{*}}$ in LSC/K$\mathrm{MoSe}_{2}$ is associated with the increased number of states near the energy of normal hydrogen electrode (NHE) potential (Supplementary Fig. 17), thus increasing the interactions between the hydrogen s orbital and the 1T- $-\mathrm{MoSe}_{2}$ states.

Excellent OER performance in $\mathrm{LSC} / \mathrm{K}-\mathrm{MoSe}_{2}$ was also explained through atomic-level simulations. Figure $4 \mathrm{f}-\mathrm{h}$ shows the free energy diagrams for OER in LSC, LSC/K-MoSe 2 , and LSC/MoSe ${ }_{2}$, respectively. Our DFT calculations strongly suggest that the OER reactions proceed with the lattice-oxygen participation mechanism ${ }^{54,55}$ dominantly rather than the conventional adsorbate evolving mechanism in the LSC/K-MoSe ${ }_{2}$ system (Supplementary Fig. 19), and the free energy barriers of reactiondetermining steps were evaluated to be $2.53,2.06$, and $1.83 \mathrm{eV}$ for LSC, LSC/MoSe $e_{2}$, and $\mathrm{LSC} / \mathrm{K}-\mathrm{MoSe}_{2}$, respectively. Taking previously reported DFT-calculated OER free energy barriers (2.05 $-2.19 \mathrm{eV})^{56-58}$ in $\mathrm{IrO}_{2}$ catalysts into account, the order of computed free energy barriers (LSC $>\mathrm{IrO}_{2}>\mathrm{LSC} / \mathrm{K}-\mathrm{MoSe}_{2}$ ) agrees well with the experimental results where the OER onset potentials decrease according to same descending order of $\mathrm{LSC}>\mathrm{IrO}_{2}>\mathrm{LSC} /$ $\mathrm{K}-\mathrm{MoSe}_{2}$, as shown in Fig. 2d. The reduced free energy barrier in the $\mathrm{LSC} / \mathrm{K}-\mathrm{MoSe}_{2}$ originates from the well-balanced free energies between two governing reactions: (1) $\mathrm{OH}^{*}$ to $\left(\mathrm{V}_{\mathrm{O}}+\mathrm{OO}\right)^{*}+\mathrm{H}^{+}$ $+e^{-}$and $(2)\left(\mathrm{H}_{\mathrm{O}}+\mathrm{OH}\right)^{*}$ to $\mathrm{OH}^{*}+\mathrm{H}^{+}+e^{-}$, where $\mathrm{OH}^{*},\left(\mathrm{~V}_{\mathrm{O}}\right.$ $+\mathrm{OO})^{*}$, and $\left(\mathrm{H}_{\mathrm{O}}+\mathrm{OH}\right)^{*}$ indicate $\mathrm{OH}$ absorbed on LSC surface, OO adsorbed on LSC surface with a neighboring oxygen vacancy, and $\mathrm{OH}$ adsorbed on LSC surface with a $\mathrm{H}$ atom adsorbed on a neighboring lattice $\mathrm{O}$ atom, respectively (see Supplementary Fig. 20 for the detailed atomic structures). While the free energy changes in reaction (1) (the red dotted circle) decrease on the order of LSC $(2.53 \mathrm{eV})>\mathrm{LSC} / \mathrm{K}-\mathrm{MoSe}_{2}(1.83 \mathrm{eV})>\mathrm{LSC} / \mathrm{MoSe}_{2}(0.67 \mathrm{eV})$, those in reaction (2) (the violet dotted circle) increase in the opposite order; LSC $(0.99 \mathrm{eV})<\mathrm{LSC} / \mathrm{K}-\mathrm{MoSe}_{2}(1.39 \mathrm{eV})<\mathrm{LSC} / \mathrm{MoSe}_{2}$ $(2.06 \mathrm{eV})$, effectively producing the lowest free energy barrier in $\mathrm{LSC} / \mathrm{K}-\mathrm{MoSe}_{2}$ with a remarkably ideal balance between the ratedetermining steps of reactions (1) and (2). Reaction (1) involves the replacement of the hydrogen in the $\mathrm{OH}$ adsorbate with the lattice oxygen that escapes from the lattice, leaving an oxygen vacancy. Therefore, the free energy barrier in reaction (1) is strongly affected by the energy difference between $\mathrm{O}^{*}$ and $\left(\mathrm{V}_{\mathrm{O}}+\right.$ $\mathrm{OO})^{*}$ structures $^{59}$ as evaluated in Supplementary Fig. 21, wherein $(\mathrm{Vo}+\mathrm{OO})^{*}$ structures are energetically more unfavorable in the same order with the free energy barriers as that in reaction (1); see DOS plots in Supplementary Fig. 22 for additional information on the instability of $\left(\mathrm{V}_{\mathrm{O}}+\mathrm{OO}\right)^{*}$ in LSC. In the case of reaction (2), the free energy barrier is related to how easily a hydrogen atom can be detached from the lattice-oxygen atom; see Supplementary Fig. 23, wherein the energy difference between $\left(\mathrm{H}_{\mathrm{O}}+\mathrm{OH}\right)^{*}$ and $\mathrm{OH}^{*}$ increases on the same order of the free energy barriers as that in reaction (2).

Overall water electrolysis of the LSC/K-MoSe ${ }_{2}$ couple. The developed LSC/K-MoSe $e_{2}$ demonstrated excellent intrinsic bifunctional catalytic activity in alkaline electrolytes for both HER and OER. Herein, the overall water electrolysis of the LSC/K$\mathrm{MoSe}_{2}$ configuration (i.e., LSC/K-MoSe ${ }_{2}$ used for both cathode and anode, denoted as LSC/K-MoSe ${ }_{2} \mid \mathrm{LSC} / \mathrm{K}-\mathrm{MoSe}_{2}$ ) was examined to further demonstrate the overall water-splitting performance and stability of $\mathrm{LSC} / \mathrm{K}-\mathrm{MoSe}_{2}$ as the bifunctional electrocatalyst in $\mathrm{N}_{2}$-saturated $1 \mathrm{M} \mathrm{KOH}$ solution. Efficient generation of the hydrogen (cathode) and oxygen (anode) gases using the LSC/K-MoSe || $\mathrm{LSC} / \mathrm{K}-\mathrm{MoSe}_{2}$ couple was confirmed, as illustrated in Fig. 5a and Supplementary Movie 1. Figure 5b shows the cell voltage $\left(E_{\text {cell }}=E_{\text {anode }}-E_{\text {cathode }}\right)$ measurement results of $\mathrm{Pt} / \mathrm{C} \| \mathrm{IrO}_{2}\left(\mathrm{Pt} / \mathrm{C}\right.$ for cathode and $\mathrm{IrO}_{2}$ for the anode) and $\mathrm{LSC} / \mathrm{K}-$ $\mathrm{MoSe}_{2} \| \mathrm{LSC} / \mathrm{K}-\mathrm{MoSe}_{2}$ obtained during the water electrolysis reaction. Consistent with the result of the half-cell-configured polarization profiles, the $\mathrm{LSC} / \mathrm{K}-\mathrm{MoSe}_{2} \mid \mathrm{LSC} / \mathrm{K}-\mathrm{MoSe}_{2}$ couple demonstrated better overall water electrolysis performance than the state-of-the-art noble-metal-based $\mathrm{Pt} / \mathrm{C} \| \mathrm{IrO}_{2}$ couple. The cell voltages needed to attain 10 and $100 \mathrm{~mA} \mathrm{~cm}^{-2}$ were 1.59 and $1.95 \mathrm{~V}$ for $\mathrm{LSC} / \mathrm{K}-\mathrm{MoSe}_{2}|| \mathrm{LSC} / \mathrm{K}-\mathrm{MoSe}_{2}$ and 1.67 and $2.04 \mathrm{~V}$ for $\mathrm{Pt} / \mathrm{C} \| \mathrm{IrO}_{2}$. Although the HER performance of $\mathrm{LSC} / \mathrm{K}-\mathrm{MoSe}_{2}$ in the half-cell reaction was slightly lower than that of $\mathrm{Pt} / \mathrm{C}$, the overwhelmingly high OER performance resulted in excellent overall water electrolysis activity for LSC/K-MoSe ${ }_{2}$, surpassing that of the noble-metal pair $\mathrm{Pt} / \mathrm{C} \| \mathrm{IrO}_{2}$. In addition to performance, electrochemical stability is an equally important criteria to consider when promoting the broad industrial pertinence of water electrolysis catalysts. To examine the electrochemical stability of $\mathrm{LSC} / \mathrm{K}-\mathrm{MoSe}_{2}$, its chronopotentiometric profile was measured at a high current density of $100 \mathrm{~mA} \mathrm{~cm}^{-2}$. As shown in Fig. $5 c$, for the $\mathrm{Pt} / \mathrm{C} / \mid \mathrm{IrO}_{2}$ reference, a rapid increase in cell voltage, which indicates cell failure, was observed within $60 \mathrm{~h}$ under our experimental test conditions. However, the LSC/K-MoSe ${ }_{2} \|$ $\mathrm{LSC} / \mathrm{K}_{-} \mathrm{MoSe}_{2}$ couple exhibited exceptionally high electrochemical durability even after $2,500 \mathrm{~h}$ of continuous operation without noticeable performance degradation. To verify the excellent operational durability of $\mathrm{LSC} / \mathrm{K}-\mathrm{MoSe}_{2}$ as the electrocatalyst, its physical and chemical characteristics were investigated after $2,500 \mathrm{~h}$ of stability testing. Supplementary Fig. 24 shows the SEM image of the catalyst electrode before and after the stability test. Even after 2,500 $\mathrm{h}$ of the electrocatalytic reaction, the starting electrode structure was well preserved without any significant physical damages or detachment. XPS analysis further reveals the superior chemical stability of LSC/K-MoSe ${ }_{2}$ (Supplementary Fig. 25 and Supplementary Tables 7, 8). After 2,500 h of 
a

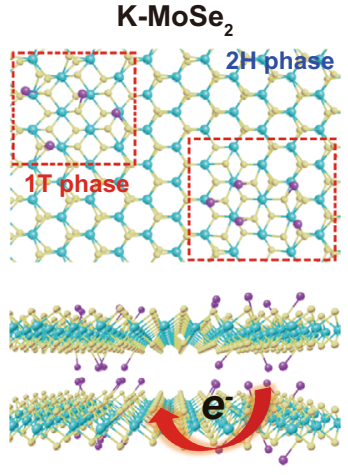

\section{LSC/K-MoSe}

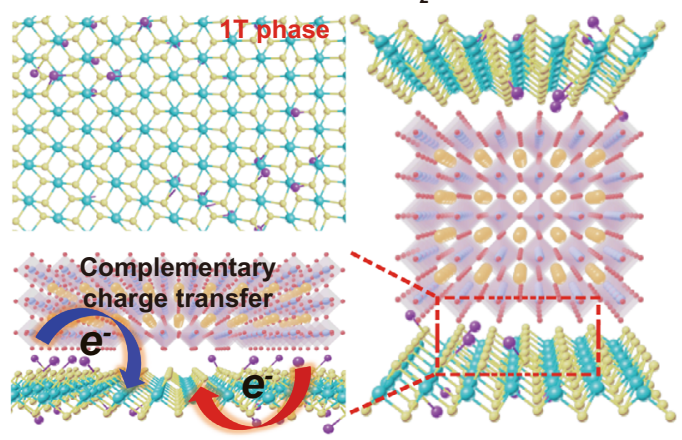

b

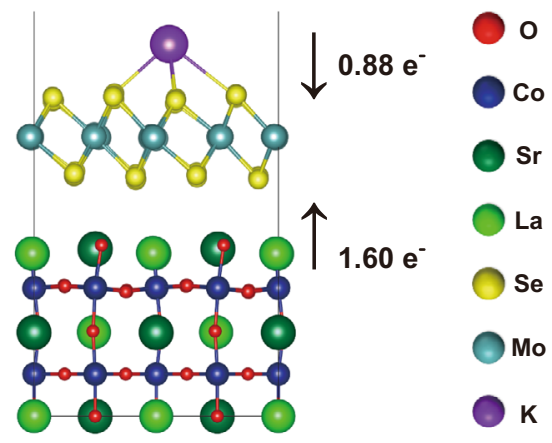

C
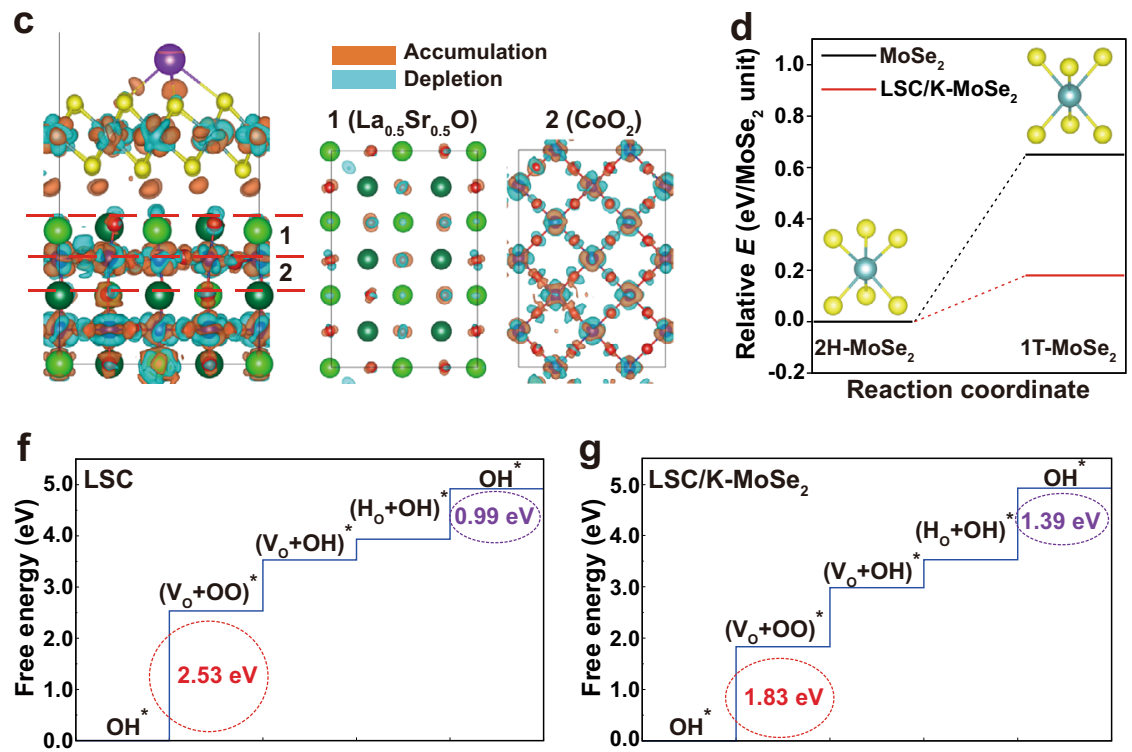

Fig. 4 Complementary charge transfer phenomena in bifunctional $\mathbf{L S C} / \mathbf{K}-\mathbf{M o S e}_{\mathbf{2}}$ catalysts. a Schematic of the atomic structure and charge transfer effect for $\mathrm{K}-\mathrm{MoSe}_{2}$ and $\mathrm{LSC} / \mathrm{K}-\mathrm{MoSe}_{2}$. Complementary charge transfer in $\mathrm{LSC} / \mathrm{K}-\mathrm{MoSe}_{2}$ can modulate the electronic structure of $\mathrm{MoSe}_{2}$, increasing the $1 \mathrm{~T}$ $\mathrm{MoSe}_{2}$ ratio in the heterostructure. b Charge transfer from K and LSC to $\mathrm{MoSe}_{2}$ in the optimized LSC/K-MoSe 2 heterostructure. c Charge density difference plot for the interface between $\mathrm{LSC}$ and K-MoSe$e_{2}$. Side view of the total structure (left) and the cross section of the layers of $\mathrm{La}_{0.5} \mathrm{Sr}_{0.5} \mathrm{O}$ (middle) and $\mathrm{CoO}_{2}$ (right) are demonstrated. $\mathbf{d}$ Relative energies of $1 \mathrm{~T}-\mathrm{MoSe}_{2}$ in monolayer structure (black line) and $\mathrm{LSC} / \mathrm{K}-\mathrm{MoSe}_{2}$ heterostructure (red line) compared to the energy of the $2 \mathrm{H}-\mathrm{MoSe}_{2}$ monolayer. e Free energy diagrams for HER for LSC, $2 \mathrm{H}-\mathrm{MoSe}_{2}, \mathrm{~K}-\mathrm{MoSe}_{2}$, and LSC/K-MoSe 2 . f-h Free energy diagrams for OER for LSC, LSC/K-MoSe 2 , and LSC/MoSe 2 .

overall water electrolysis reaction, each ratio of the $\mathrm{Co}^{3+} / \mathrm{Co}^{2+}$ in Co $2 p$ and surface-active oxygen/lattice oxygen in $\mathrm{O} 1 \mathrm{~s}$ exhibited almost negligible changes compared with those of pristine LSC/K$\mathrm{MoSe}_{2}$. MoSe 2 may potentially be decomposed into molybdenum oxides and selenate at the potentials of the OER electrode during prolonged electrolysis ${ }^{60}$. To verify the stability of $\mathrm{MoSe}_{2}$ in LSC/ $\mathrm{K}-\mathrm{MoSe}_{2}$, we performed XPS analysis for Mo and Se in LSC/K$\mathrm{MoSe}_{2}$ on the OER electrode after 2,500 h of chronopotentiometric stability test. Although some partial oxidization was observed, the integrity of $\mathrm{MoSe}_{2}$ was well preserved without decomposition (Supplementary Fig. 26 and Supplementary Table 9). Moreover, the energy efficiency of the overall water electrolysis using LSC/K-MoSe $\mathrm{K}_{2} \| \mathrm{LSC} / \mathrm{K}-\mathrm{MoSe}_{2}$ at $100 \mathrm{~mA} \mathrm{~cm}{ }^{-2}$ was calculated to be $75.4 \%$ (Supplementary Note 1). Considering the energy efficiency of a typical noble-metal-based water electrolysis catalyst is around $70 \%$, our results demonstrate that the developed bifunctional $\mathrm{LSC} / \mathrm{K}-\mathrm{MoSe}_{2}$ catalyst can be used as a promising electrocatalyst in water electrolysis for efficient hydrogen production. Figure 5d (also summarized in Supplementary Table 10) compares the electrochemical stability of overall water electrolysis for various catalyst configurations reported to date along with the proposed $\mathrm{LSC} / \mathrm{K}-\mathrm{MoSe}_{2}$ in this work. Additionally, we further investigated the operational durability of the LSC/K-MoSe $e_{2}$ in various harsh environments. Chronopotentiometric stability test of the LSC/K-MoSe $e_{2}$ couple was conducted under high operational temperature and current density $\left(500\right.$ and $1,000 \mathrm{~mA} \mathrm{~cm}^{-2}$ in $1 \mathrm{M} \mathrm{KOH}$ at $\left.60{ }^{\circ} \mathrm{C}\right)$ and high electrolyte concentration $\left(100 \mathrm{~mA} \mathrm{~cm}^{-2}\right.$ in $10 \mathrm{M}$ $\mathrm{KOH}$ at room temperature). In $1 \mathrm{M} \mathrm{KOH}$ at $60^{\circ} \mathrm{C}$, the $\mathrm{LSC} / \mathrm{K}$ $\mathrm{MoSe}_{2}$ couple required cell voltages of $2.25 \mathrm{~V}$ at $500 \mathrm{~mA} \mathrm{~cm}^{-2}$ and $2.52 \mathrm{~V}$ at $1000 \mathrm{~mA} \mathrm{~cm}^{-2}$, respectively (Supplementary Fig. 27). Under such conditions, it exhibited stable operational stability over 1,200 and $800 \mathrm{~h}$ at 500 and $1,000 \mathrm{~mA} \mathrm{~cm}^{-2}$, respectively, without obvious performance degradation (Supplementary Fig. 28). In $10 \mathrm{M} \mathrm{KOH}$ at room temperature, the cell voltage needed to achieve $100 \mathrm{~mA} \mathrm{~cm}^{-2}$ was $1.87 \mathrm{~V}$ for the LSC/K-MoSe couple (Supplementary Fig. 29). In the two-electrode cell, the chronopotentiometric stability of $1,600 \mathrm{~h}$ was achieved at $100 \mathrm{~mA}$ $\mathrm{cm}^{-2}$ in $10 \mathrm{M} \mathrm{KOH}$ at room temperature without any noticeable performance degradation (Supplementary Fig. 30). Despite the various accelerated test conditions, our heterostructure demonstrates overwhelmingly superior durability for water electrolysis. 
a
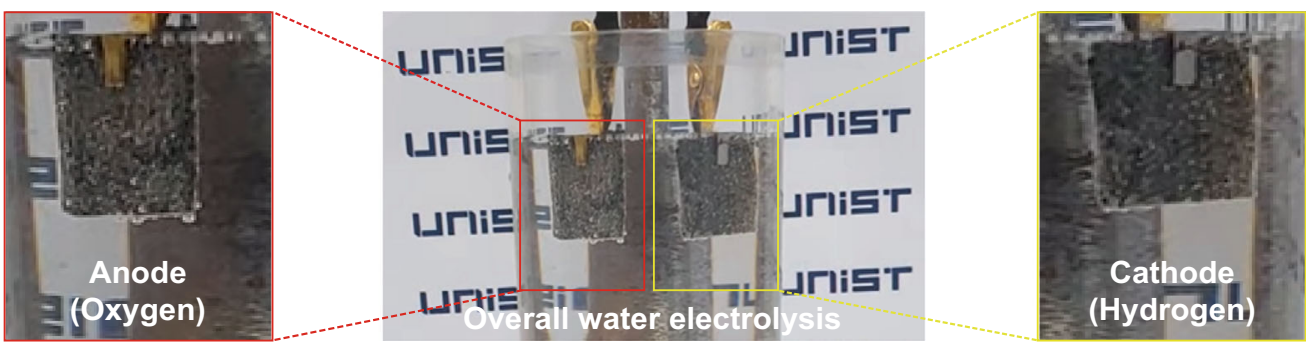

b

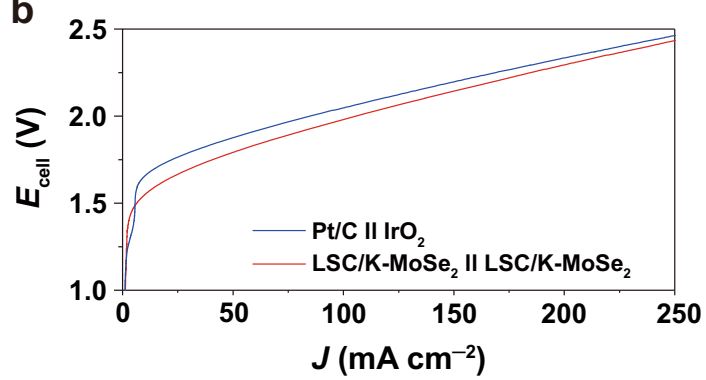

C

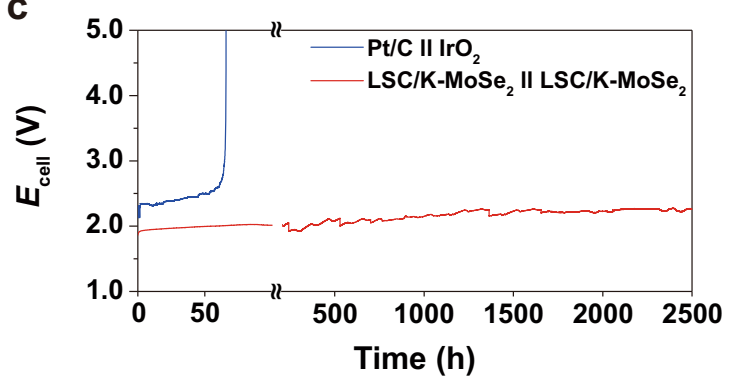

d

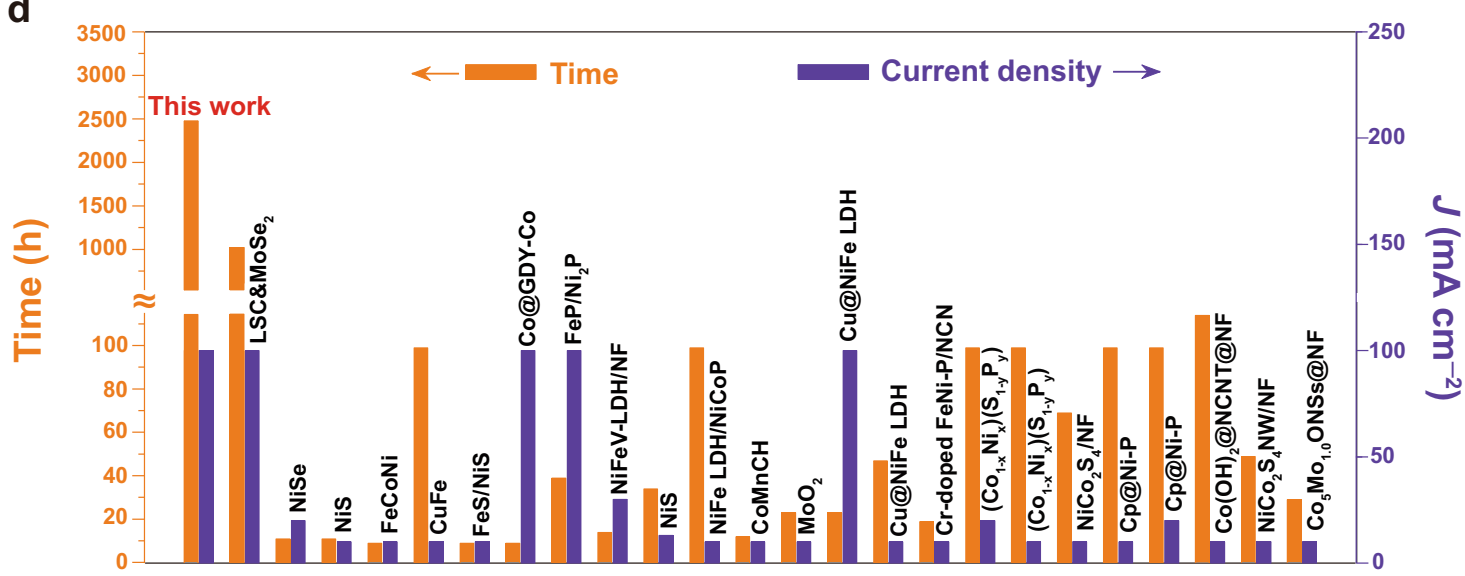

Fig. 5 Overall water electrolysis of $\mathbf{L S C} / \mathbf{K}-\mathbf{M o S e}_{2}$. a Digital image of full-cell water-splitting system comprising a two-electrode configuration with LSC/K-

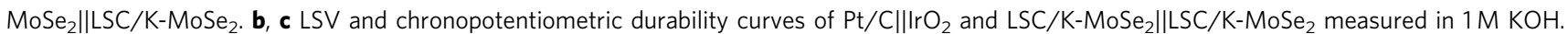
d Summary of the overall water electrolysis stability of $\mathrm{LSC} / \mathrm{K}-\mathrm{MoSe}_{2} \| \mathrm{LSC} / \mathrm{K}-\mathrm{MoSe}_{2}$ and other reported electrocatalyst couples.

\section{Discussion}

In this work, we developed a heterostructure-based electrocatalyst that demonstrates excellent overall water electrolysis performance and stability. The complementary charge transfer induced within the heterogeneous catalyst, comprising alkalimetal-treated transition-metal dichalcogenides and perovskite oxide, generated synergistic effects in both HER and OER processes. The modulated electronic structure of $\mathrm{MoSe}_{2}$ with high metallic-phase purity and improved electrical conductivity enhanced HER kinetics. The increased electrophilicity of LSC improved the adsorption capability of oxygen-generating intermediates on the catalyst surface, thereby boosting OER performance over that of $\mathrm{IrO}_{2}$. The water electrolysis performance using the $\mathrm{LSC} / \mathrm{K}-\mathrm{MoSe}_{2}|| \mathrm{LSC} / \mathrm{K}-\mathrm{MoSe}_{2}$ couple outperformed the state-of-the-art noble-metal pair of $\mathrm{Pt} / \mathrm{C} \| \mathrm{IrO}_{2}$, exhibiting lower cell voltage for the overpotential at 10 and 100 $\mathrm{mA} \mathrm{cm}{ }^{-2}$, improved energy efficiency, and excellent operational stability over $2,500 \mathrm{~h}$. This work can provide a promising perspective for the performance maximization of heterostructurebased catalysts in water electrolysis to substitute precious-metalbased electrocatalysts.

\section{Methods}

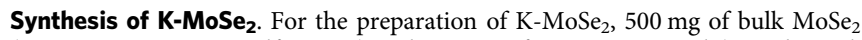
$(<2 \mu \mathrm{m}$, purity $>99 \%$, Alfa Aesar) and $200 \mathrm{mg}$ of potassium metal (stored in oil, purity $>99.95 \%$, Kojundo, Korea) were added to a glass tube under inert conditions in a glovebox. The tube was sealed and treated at $400^{\circ} \mathrm{C}$ for $1 \mathrm{~h}$. The as-prepared potassium-intercalated $\mathrm{MoSe}_{2}$ was rinsed with deionized water and ethanol to remove potassium ion residues. The resulting powder was dried at $80^{\circ} \mathrm{C}$ for $24 \mathrm{~h}$.

Synthesis of LSC. To synthesize the LSC perovskite oxides, an aqueous solution containing dissolved $\mathrm{La}, \mathrm{Sr}$, and Co nitrites $\left(\mathrm{La}\left(\mathrm{NO}_{3}\right)_{3} \cdot 6 \mathrm{H}_{2} \mathrm{O}, \mathrm{Sr}\left(\mathrm{NO}_{3}\right)_{2}, \mathrm{Co}\left(\mathrm{NO}_{3}\right)_{2}\right.$. $6 \mathrm{H}_{2} \mathrm{O}$, Alfa Aesar) in stoichiometric amounts and citric acid $\left(\mathrm{C}_{6} \mathrm{H}_{8} \mathrm{O}_{7}\right.$, Sigma-Aldrich) in deionized water was prepared. Post solvent evaporation, the resulting wet-gel was calcined at $900^{\circ} \mathrm{C}$ for $2 \mathrm{~h}$ and at $950^{\circ} \mathrm{C}$ for $10 \mathrm{~h}$ to remove the organic fraction. Finally, the resulting reaction product was mortared to homogenize the LSC.

Synthesis of LSC/K-MoSe $e_{2}$. To synthesize the LSC/K-MoSe $e_{2}$ heterostructure, as prepared LSC and $\mathrm{K}-\mathrm{MoSe}_{2}$ were high-energy milled with 10 wt.\% of Ketjen Black (KB) (EC-600JD, Lion Specialty Chemicals Co. Ltd.) via the planetary ball mill system (PM-100, Retsch). To find the optimized weight ratio for LSC and K-MoSe for electrochemical performance, various mixture weight ratios were examined with LSC:K-MoSe $2: \mathrm{KB}$ of 8:1:1, 7:2:1, 6:3:1, and 5:4:1. The total weight of the mixture was maintained as $600 \mathrm{mg}$. LSC, $\mathrm{K}-\mathrm{MoSe}_{2}$, and $\mathrm{KB}$ in ethanol were sealed into the steel jar and ball-milled at $500 \mathrm{rpm}$ for $2 \mathrm{~h}$. After completing the ballmilling process, the resulting product was thoroughly dried and collected. 
Material characterizations. The morphology and EDS elemental mapping of the catalysts were characterized using HR-TEM (JEM-2100F, JEOL) with an accelerating voltage of $200 \mathrm{kV}$. Crystallographic information of catalysts was analyzed through highpower XRD (D/MAX2500V/PC, Rigaku) at $40 \mathrm{kV}$ and $200 \mathrm{~mA}$ at a scanning rate of $1^{\circ}$ $\mathrm{min}^{-1}$ in the diffraction range of $10^{\circ}-80^{\circ}$. The chemical state and work function were investigated via XPS (ESCALAB 250XI, Thermo Fisher Scientific) with monochromated $\mathrm{Al}-\mathrm{Ka}$ radiation. TGA analysis was conducted to investigate the surface adsorption capacity of catalysts at a ramping temperature rate of $10^{\circ} \mathrm{C} \mathrm{min}^{-1}$ using thermogravimetric analyzer $(\mathrm{Q} 500, \mathrm{TA})$. The surface area and pore size of the catalysts were evaluated using $\mathrm{N}_{2}$ desorption/adsorption isotherms of catalysts using a physisorption analyzer (ASAP 2420, Micromeritics Instruments). The optical properties of the catalysts were collected using a UV-Vis-NIR spectrophotometer (Cary 5000, Agilent). Raman spectra were recorded using confocal Raman spectroscopy (Alpha300R, WITec) equipped with a 532-nm laser. The electrode morphologies before and after overall water electrolysis were obtained via cold FE-SEM (S-4800, HITACHI).

Electrochemical measurements. Half-cell electrochemical measurements were performed in $1 \mathrm{M} \mathrm{KOH}$, in which saturated $\mathrm{Ag} / \mathrm{AgCl}$ and a carbon rod were used as the reference and counter electrodes, respectively, in a three-electrode configuration controlled by an electrochemical workstation (CHI 760E, CH Instruments Inc.). Catalyst ink including LSC/K-MoSe 2 , K-MoSe $e_{2}$, and LSC was prepared by dispersing $9 \mathrm{mg}$ of the catalyst and $1 \mathrm{mg}$ of $\mathrm{KB}$ in a $1 \mathrm{~mL}$ binder solution comprising 5 wt.\% Nafion solution (Sigma-Aldrich), ethanol, and isopropyl alcohol, followed by bath sonication. A catalyst ink of $\mathrm{Pt} / \mathrm{C}$ and $\mathrm{IrO}_{2}$ was similarly prepared except for the $\mathrm{KB}$. The working electrode was prepared via drop-casting $5 \mu \mathrm{L}$ of the as-prepared catalyst inks onto the glassy carbon disk electrode with an area of $0.071 \mathrm{~cm}^{2}$. The HER and OER LSV polarization curves were obtained at a scan rate of $5 \mathrm{mV} \mathrm{s}^{-1}$ in $\mathrm{N}_{2}$-saturated electrolyte, which were measured from 0 to $-1.0 \mathrm{~V}$ (vs. reversible hydrogen electrode (RHE)) and from 1.0 to $2.0 \mathrm{~V}$ (vs. RHE), respectively. Tafel slope values were derived from the LSV curves by plotting the overpotential against current density in $\log$-scale from 1 to $10 \mathrm{~mA} \mathrm{~cm}^{-2}$. All potentials in this work were measured with respect to $\mathrm{Ag} / \mathrm{AgCl}$ reference electrode and converted to RHE scale using the following formula in $1 \mathrm{M} \mathrm{KOH} \mathrm{(pH} \mathrm{14):} E$ (vs. $\mathrm{RHE})=E(\mathrm{vs} . \mathrm{Ag} / \mathrm{AgCl})+E_{\mathrm{Ag} / \mathrm{AgCl}}(=0.197 \mathrm{~V})+0.0592 \mathrm{pH}=E(\mathrm{vs} . \mathrm{Ag} / \mathrm{AgCl})+$ $1.0258 \mathrm{~V}$. EIS measurements were conducted at an overpotential of -0.2 and $0.7 \mathrm{~V}$ (vs. RHE) for HER and OER, respectively, in a frequency range of $100 \mathrm{kHz}$ to $0.01 \mathrm{~Hz}$ with an amplitude of $10 \mathrm{mV}$ in $1 \mathrm{M} \mathrm{KOH}$. All half-cell polarization curves were corrected for ohmic losses by the following equation. $E=E(\mathrm{RHE})-i \mathrm{R}_{\mathrm{s}}$, where $E$ is the potential after the $i R$-correction, $E(\mathrm{RHE})$ is the measured potential with respect to RHE (before $i R$ correction), $i$ is the measured current, and $R_{\mathrm{s}}$ is the uncompensated resistance obtained from EIS analysis. To measure the double-layer capacitance $\left(C_{\mathrm{dl}}\right)$ value, the potential window of cyclic voltammograms was cycled in the non-Faradaic region from 0.03 to $0.33 \mathrm{~V}$ (vs. RHE) with different scan rates from 20 to $160 \mathrm{mV} \mathrm{s}^{-1}$. $C_{\mathrm{dl}}$ values were derived by plotting the charging current density difference $\left(\Delta j=\left(j_{\mathrm{a}}-j_{\mathrm{c}}\right) / 2\right)$ at $0.18 \mathrm{~V}$. Overall water electrolysis testing was conducted using a two-electrode configuration comprising electrosprayed catalyst inks on $\mathrm{Ni}$ foam (with a catalyst loading of $1 \mathrm{mg} \mathrm{cm}$ ${ }^{-2}$ ) as the current collector. Chronopotentiometry stability test results of the overall water electrolysis were obtained under a current density of $100 \mathrm{~mA} \mathrm{~cm}^{-2}$ using an electrochemical workstation (ZIVE BP2C, Wonatech Co., Ltd.).

Computational details. We performed spin-polarized ab initio calculations using the Vienna ab initio simulation package (VASP) ${ }^{61}$ within the projector augmented wave (PAW) method ${ }^{62}$ and Perdew-Burke-Ernzerhof (PBE) ${ }^{63}$ exchange and correlation functionals. The DFT $+U$ method based on Dudarev's approach ${ }^{64}$ was adopted with $U=4.3$ and $J=1.0 \mathrm{eV}\left(U_{\text {eff }}=3.3 \mathrm{eV}\right)$ for Co-3d and $U_{\text {eff }}=4.0 \mathrm{eV}$ for Mo- $4 d$, as employed in previous studies ${ }^{65,66}$. First, the LSC slab structure was prepared based on $2 \sqrt{2} \times 3 \sqrt{2} \times 2$ supercells with $>20 \AA$ vacuum space. A $1 \times 1 \times 1$ Monkhorst-pack $k$-points mesh was adopted with $2 \times 10^{-2} \mathrm{eV}^{-1}$ for force criterion in the ionic relaxations and a $400 \mathrm{eV}$ energy cutoff for the plane-wave basis set was used with valance electron configurations of $5 s^{2} 5 p^{6} 5 d^{1} 6 s^{2}(\mathrm{La}), 4 s^{2} 4 p^{6} 5 s^{2}$ (Sr_sv), $3 d^{8} 4 s^{1}(\mathrm{Co}), 2 s^{2} 2 p^{4}(\mathrm{O}), 4 s^{2} 4 p^{6} 4 d^{5} 5 s^{1}$ (Mo_sv), $4 s^{2} 4 p^{4}$ (Se), and $3 s^{2} 3 p^{6} 4 s^{1}$ (K_sv) orbitals for La, Sr, Co, O, Mo, Se, and K, respectively. The LSC/MoSe 2 and $\mathrm{LSC} / \mathrm{K}-\mathrm{MoSe}_{2}$ structures were modeled using $2 \times 5$ supercell of $2 \mathrm{H}-$ or $1 \mathrm{~T}-\mathrm{MoSe}_{2}$ monolayer placed on the LSC (001) surfaces with $<3 \%$ lattice mismatch for LSC/K$\mathrm{MoSe}_{2}$ (the length of the cell-vectors: $a=11.20 \AA, b=16.57 \AA$, and $c=35.00 \AA$ ), $\mathrm{K}$ atoms were attached on the $\mathrm{MoSe}_{2}$ monolayer at the energetically most stable site, where one $\mathrm{K}$ atom was adsorbed on the $2 \times 5 \mathrm{MoSe}_{2}$ supercell surface (Fig. $4 \mathrm{~b}$ ). The top two atomic layers of LSC slab structure were relaxed to simulate the heterostructures. The charge transfer in $\mathrm{LSC} / \mathrm{K}-\mathrm{MoSe}_{2}$ structure was analyzed using Bader population analysis ${ }^{50}$. Second, the free energy of HER reactions was computed until the residual force components were within $5 \times 10^{-3} \mathrm{eV} \AA^{-1}$ using the equation $G_{\mathrm{H}}=E(\mathrm{H})+0.24 \mathrm{eV}$, wherein $E(\mathrm{H})$ is the adsorption energy of a $\mathrm{H}$ atom, calculated for $1 / 2 \mathrm{H}_{2}$ at $\mathrm{pH}=0$ and $p\left(\mathrm{H}_{2}\right)=1 \mathrm{bar}$, and $0.24 \mathrm{eV}$ correction is for the differences in zero-point-energy and entropy ${ }^{67,68}$. The free energy of each subreaction for OER was evaluated using the equation $G=\Delta E+\Delta \mathrm{ZPE}-T \Delta S$ at $\mathrm{pH}=0, T=298 \mathrm{~K}$, and zero applied potential ( $0 \mathrm{~V}$ vs. RHE), where $\Delta G, \Delta E$, $\Delta \mathrm{ZPE}, T$, and $\Delta S$ represent the change in free energy, total energy difference, change in zero-point-energy, temperature, and change in entropy, respectively. The $\triangle Z P E$ and $T \Delta S$ terms were adopted from previous studies and gas phase $\mathrm{H}_{2} \mathrm{O}$ and $\mathrm{O}_{2}$ were considered references for all the reactions, assuming 0.035 bar $\mathrm{H}_{2} \mathrm{O}$ gas pressure at room temperature for equilibrium with liquid water. The free energy $\mathrm{O}_{2}$ gas was computed by fixing the free energy changes in the overall reaction $\left(\mathrm{H}_{2} \mathrm{O} \rightarrow 1 / 2 \mathrm{O}_{2}+\mathrm{H}_{2}\right)$ to the experimentally measured value, $2.46 \mathrm{eV}$, as adopted in previously reported OER computations ${ }^{59,69-73}$. To model OER on LSC (001) surfaces in LSC/K-MoSe 2 , we used the cropped $2 \times 1.5 \mathrm{MoSe}_{2}$ supercells to expose the LSC (001) surface to secure enough space for OER adsorbates on the LSC surface. A $4 \times 4 \times 1$ Monkhorst-pack $k$-points mesh was used to analyze the DOS.

\section{Data availability}

The data measured, simulated, and analyzed in this study are available from the corresponding author on reasonable request.

Received: 14 January 2021; Accepted: 9 July 2021; Published online: 29 July 2021

\section{References}

1. Seh, Z. W. et al. Combining theory and experiment in electrocatalysis: Insights into materials design. Science 355, 6321 (2017).

2. You, B. et al. Enhancing electrocatalytic water splitting by strain engineering. Adv. Mater. 31, 1807001 (2019)

3. Cheng, N. et al. Platinum single-atom and cluster catalysis of the hydrogen evolution reaction. Nat. Commun. 7, 1-9 (2016)

4. Yu, F. et al. High-performance bifunctional porous non-noble metal phosphide catalyst for overall water splitting. Nat. Commun. 9, 2551 (2018).

5. Jiao, Y. et al. Design of electrocatalysts for oxygen-and hydrogen-involving energy conversion reactions. Chem. Soc. Rev. 44, 2060-2086 (2015).

6. Liang, C. et al. Exceptional performance of hierarchical Ni-Fe oxyhydroxide@NiFe alloy nanowire array electrocatalysts for large current density water splitting. Energy Environ. Sci. 13, 86-95 (2020).

7. Huang, H. et al. Rapid and energy-efficient microwave pyrolysis for high-yield production of highly-active bifunctional electrocatalysts for water splitting. Energy Environ. Sci. 13, 545-553 (2020).

8. McCrory, C. C. et al. Benchmarking heterogeneous electrocatalysts for the oxygen evolution reaction. J. Am. Chem. Soc. 135, 16977-16987 (2013).

9. Liang, Y. et al. Strongly coupled inorganic/nanocarbon hybrid materials for advanced electrocatalysis. J. Am. Chem. Soc. 135, 2013-2036 (2013).

10. Zhang, T. et al. Heteroatom-doped carbon materials for hydrazine oxidation. Adv. Mater. 31, 1804394 (2019).

11. Zhu, Q. L. et al. Controlled synthesis of ultrafine surfactant-free NiPt nanocatalysts toward efficient and complete hydrogen generation from hydrazine borane at room temperature. ACS Catal. 4, 4261-4268 (2014).

12. Zhao, P. et al. NiIr nanoparticles immobilized on the pores of MIL-101 as highly efficient catalyst toward hydrogen generation from hydrous hydrazine. ACS Sustain. Chem. Eng. 3, 1086-1093 (2015).

13. Kang, Y. et al. Bimetallic AuRh nanodendrites consisting of Au icosahedron cores and atomically ultrathin Rh nanoplate shells: synthesis and light-enhanced catalytic activity. NPG Asia Mater. 9, e407 (2017).

14. Oh, N. K. et al. In-situ local phase-transitioned $\mathrm{MoSe}_{2}$ in $\mathrm{La}_{0.5} \mathrm{Sr}_{0.5} \mathrm{CoO}_{3-\delta}$ heterostructure and stable overall water electrolysis over $1000 \mathrm{~h}$. Nat. Commun. 10, 1723 (2019)

15. Sarkar, S. et al. Stress-induced electronic structure modulation of manganeseincorporated $\mathrm{Ni}_{2} \mathrm{P}$ leading to enhanced activity for water splitting. ACS Appl. Energy Mater. 3, 1271-1278 (2020)

16. Fu, Q. et al. 2D Transition metal dichalcogenides: design, modulation, and challenges in electrocatalysis. Adv. Mater. 33, 1907818 (2021).

17. Huang, J. et al. Cytomembrane-structure-inspired active $\mathrm{Ni}-\mathrm{N}-\mathrm{O}$ interface for enhanced oxygen evolution reaction. Adv. Mater. 30, 1803367 (2018).

18. Yao, Y. et al. Engineering the electronic structure of single atom Ru sites via compressive strain boosts acidic water oxidation electrocatalysis. Nat. Catal. 2, 304-313 (2019).

19. Tang, C. et al. NiSe nanowire film supported on nickel foam: an efficient and stable 3D bifunctional electrode for full water splitting. Angew. Chem. Int. Ed. 127, 9483-9487 (2015).

20. Xing, J. et al. Electro-synthesis of 3D porous hierarchical Ni-Fe phosphate film/Ni foam as a high-efficiency bifunctional electrocatalyst for overall water splitting. J. Mater. Chem. A 4, 13866-13873 (2016).

21. Yang, Y. et al. Tuning electronic structures of nonprecious ternary alloys encapsulated in graphene layers for optimizing overall water splitting activity. ACS Catal. 7, 469-479 (2017).

22. Inamdar, A. I. et al. A robust nonprecious CuFe composite as a highly efficient bifunctional catalyst for overall electrochemical water splitting. Small 16, 1905884 (2020).

23. Zhang, R. et al. Hydrolysis assisted in-situ growth of 3D hierarchical FeS/NiS/ nickel foam electrode for overall water splitting. Electrochim. Acta 332, 135534 (2020). 
24. Xie, J. et al. Intralayered ostwald ripening to ultrathin nanomesh catalyst with robust oxygen-evolving performance. Adv. Mater. 29, 1604765 (2017).

25. Gong, M. et al. Nanoscale nickel oxide/nickel heterostructures for active hydrogen evolution electrocatalysis. Nat. Commun. 5, 1-6 (2014).

26. Park, S. et al. Phase engineering of transition metal dichalcogenides with unprecedentedly high phase purity, stability, and scalability via molten-metalassisted intercalation. Adv. Mater. 32, 2001889 (2020).

27. Zheng, Y. et al. Hydrogen evolution by a metal-free electrocatalyst. Nat. Commun. 5, 3783 (2014).

28. Shao, F. Q. et al. One-pot synthesis of hollow AgPt alloyed nanocrystals with enhanced electrocatalytic activity for hydrogen evolution and oxygen reduction reactions. J. Colloid Interface sci. 505, 307-314 (2017).

29. Markovic, N. M. et al. Temperature-dependent hydrogen electrochemistry on platinum low-index single-crystal surfaces in acid solutions. J. Phys. Chem. B 101, 5405-5413 (1997).

30. Sun, H. et al. Molybdenum and niobium codoped B-site-ordered double perovskite catalyst for efficient oxygen evolution reaction. ACS Appl. Mater. Interfaces 10, 16939-16942 (2018).

31. Tariq, M. et al. Unraveling the beneficial electrochemistry of $\mathrm{IrO}_{2} / \mathrm{MoO}_{3}$ hybrid as a highly stable and efficient oxygen evolution reaction catalyst. ACS Sustain. Chem. Eng. 6, 4854-4862 (2018).

32. $\mathrm{Li}, \mathrm{X}$. et al. Crystallographic-orientation dependent $\mathrm{Li}$ ion migration and reactions in layered $\mathrm{MoSe}_{2} .2 D$ Mater. 6, 035027 (2019).

33. Hua, B. et al. A coupling for success: controlled growth of $\mathrm{Co} / \mathrm{CoO}_{\mathrm{x}}$ nanoshoots on perovskite mesoporous nanofibres as high-performance trifunctional electrocatalysts in alkaline condition. Nano Energy 32, 247-254 (2017).

34. Han, L. et al. Dealloying-directed synthesis of efficient mesoporous CoFebased catalysts towards the oxygen evolution reaction and overall water splitting. Nanoscale 9, 16467-16475 (2017).

35. Klingan, $\mathrm{K}$. et al. Water oxidation by amorphous cobalt-based oxides: volume activity and proton transfer to electrolyte bases. ChemSusChem 7, 1301-1310 (2014).

36. Gupta, U. et al. Characterization of few-layer $1 \mathrm{~T}-\mathrm{MoSe}_{2}$ and its superior performance in the visible-light induced hydrogen evolution reaction. APL Mater. 2, 092802 (2014).

37. Lu, Shi-Yu et al. Greatly boosting electrochemical hydrogen evolution reaction over $\mathrm{Ni}_{3} \mathrm{~S}_{2}$ nanosheets rationally decorated by $\mathrm{Ni}_{3} \mathrm{Sn}_{2} \mathrm{~S}_{2}$ quantum dots. Appl. Catal. B 267, 118675 (2020)

38. Brooman, E. W. et al. Correlations between the rate of the hydrogen electrode reaction and the properties of alloys. J. Electroanal. Chem. 49, 325-353 (1974).

39. Zeradjanin, A. R. et al. Balanced work function as a driver for facile hydrogen evolution reaction-comprehension and experimental assessment of interfacial catalytic descriptor. Phys. Chem. Chem. Phys. 19, 17019-17027 (2017).

40. Henckel, D. A. et al. Improved HER catalysis through facile, aqueous electrochemical activation of nanoscale WSe ${ }_{2}$. Nano Lett. 18, 2329-2335 (2018).

41. Samikannu, S. et al. Dissipative soliton generation in an all-normal dispersion ytterbium-doped fiber laser using few-layer molybdenum diselenide as a saturable absorber. Opt. Eng. 55, 081311 (2016).

42. Xia, Y. et al. Camphorsulfonic acid fully doped polyaniline emeraldine salt: conformations in different solvents studied by an ultraviolet/visible/ near-infrared spectroscopic method. Chem. Mater. 7, 443-445 (1995).

43. Suntivich, J. et al. A perovskite oxide optimized for oxygen evolution catalysis from molecular orbital principles. Science 334, 1383-1385 (2011).

44. Maitra, U. et al. Importance of trivalency and the $\mathrm{e}_{\mathrm{g}}{ }^{1}$ configuration in the photocatalytic oxidation of water by $\mathrm{Mn}$ and Co oxides. Proc. Natl Acad. Sci. USA 110, 11704-11707 (2013).

45. Wang, Q. et al. $\mathrm{La}_{0.8} \mathrm{Sr}_{0.2} \mathrm{Co}_{1-x} \mathrm{Mn}_{x} \mathrm{O}_{3}$ perovskites as efficient bi-functional cathode catalysts for rechargeable zinc-air batteries. Electrochim. Acta $\mathbf{2 5 4}$, 14-24 (2017)

46. Wang, $\mathrm{Z}$. et al. Nickel-doped $\mathrm{La}_{0.8} \mathrm{Sr}_{0.2} \mathrm{Mn}_{1-x} \mathrm{Ni}_{x} \mathrm{O}_{3}$ nanoparticles containing abundant oxygen vacancies as an optimized bifunctional catalyst for oxygen cathode in rechargeable lithium-air batteries. ACS Appl. Mater. Interfaces 8, 6520-6528 (2016).

47. Hua, B. et al. All-in-one perovskite catalyst: smart controls of architecture and composition toward enhanced oxygen/hydrogen evolution reactions. $A d v$. Energy Mater. 7, 1700666 (2017).

48. Ito, Y. et al. High catalytic activity of nitrogen and sulfur co-doped nanoporous graphene in the hydrogen evolution reaction. Angew. Chem. Int. Ed. 127, 2159-2164 (2015).

49. Kim, M. et al. Promotion of electrochemical oxygen evolution reaction by chemical coupling of cobalt to molybdenum carbide. Appl. Catal. B 227, 340-348 (2018).

50. Henkelman, G. et al. A fast and robust algorithm for Bader decomposition of charge density. Comput. Mater. Sci. 36, 354-360 (2006).

51. Hinnemann, B. et al. Biomimetic hydrogen evolution: $\mathrm{MoS}_{2}$ nanoparticles as catalyst for hydrogen evolution. J. Am. Chem. Soc. 127, 5308-5309 (2005).
52. Zheng, Y. et al. Toward design of synergistically active carbon-based catalysts for electrocatalytic hydrogen evolution. ACS Nano. 8, 5290-5296 (2014).

53. Ling, F. et al. Enhancing hydrogen evolution on the basal plane of transition metal dichacolgenide van der Waals heterostructures. Npj Comput. Mater. 5, 20 (2019).

54. Grimaud, A. et al. Activating lattice oxygen redox reactions in metal oxides to catalyse oxygen evolution. Nat. Chem. 9, 457-465 (2017).

55. Mefford, J. T. et al. Water electrolysis on $\mathrm{La}_{1-\mathrm{x}} \mathrm{Sr}_{\mathrm{x}} \mathrm{CoO}_{3-\delta}$ perovskite electrocatalysts. Nat. Commun. 7, 11053 (2016).

56. Zagalskaya, A. et al. Role of defects in the interplay between adsorbate evolving and lattice oxygen mechanisms of the oxygen evolution reaction in $\mathrm{RuO}_{2}$ and $\mathrm{IrO}_{2}$. ACS Catal. 10, 3650-3657 (2020).

57. Buvat, G. et al. OER performances of cationic substituted (100)-oriented $\mathrm{IrO}_{2}$ thin films: a joint experimental and theoretical study. ACS Appl. Energy Mater. 3, 5229-5237 (2020).

58. Briquet, L. G. V. et al. A new type of scaling relations to assess the accuracy of computational predictions of catalytic activities applied to the oxygen evolution reaction. Chem CatChem 9, 1261-1268 (2017)

59. Rong, X. et al. A fundamental relationship between reaction mechanism and stability in metal oxide catalysts for oxygen evolution. ACS Catal. 6 , 1153-1158 (2016)

60. Tang, Y. J. et al. In situ oxidation transformation of trimetallic selenide to amorphous FeCo-oxyhydroxide by self-sacrificing $\mathrm{MoSe}_{2}$ for efficient water oxidation. J. Mater. Chem. A 8, 7925-7934 (2020).

61. Kresse, G. et al. Efficient iterative schemes for ab initio total-energy calculations using a plane-wave basis set. Phys. Rev. B. 54, 11169-11186 (1996).

62. Blöchl, P. E. et al. Projector augmented-wave method. Phys. Rev. B. 50, 17953-17979 (1994)

63. Perdew, J. P. et al. Generalized gradient approximation made simple. Phys. Rev. Lett. 77, 3865-3868 (1996).

64. Dudarev, S. et al. Electron-energy-loss spectra and the structural stability of nickel oxide: An LSDA+U study. Phys. Rev. B 57, 1505-1509 (1998).

65. Grimaud, A. et al. Activating lattice oxygen redox reactions in metal oxides to catalyse oxygen evolution. Nat. Chem. 9, 457-465 (2017).

66. Guguchia, A. et al. Magnetism in semiconducting molybdenum dichalcogenides. Sci. Adv. 4, eaat3672 (2018).

67. Vikraman, D. et al. Design of basal plane edges in metal-doped nanostripesstructured $\mathrm{MoSe}_{2}$ atomic layers to enhance hydrogen evolution reaction activity. ACS Sustain. Chem. Eng. 7, 458-469 (2019).

68. Mir, S. H. et al. A comparative study of hydrogen evolution reaction on pseudo-monolayer $\mathrm{WS}_{2}$ and $\mathrm{PtS}_{2}$ : insights based on the density functional theory. Catal. Sci. Technol. 7, 687-692 (2017).

69. Yoo, J. S. et al. Role of lattice oxygen participation in understanding trends in the oxygen evolution reaction on perovskites. ACS Catal. 8, 4628-4636 (2018).

70. Man, I. C. et al. Universality in oxygen evolution electrocatalysis on oxide surfaces. ChemCatChem 3, 1159-1165 (2011).

71. Yao, Y. et al. Engineering the electronic structure of single atom Ru sites via compressive strain boosts acidic water oxidation electrocatalysis. Nat. Catal. 2, 304-313 (2019).

72. Huang, Z. F. et al. Chemical and structural origin of lattice oxygen oxidation in Co-Zn oxyhydroxide oxygen evolution electrocatalysts. Nat. Energy 4 , 329-338 (2019).

73. Seo, M. H. et al. Design of highly active perovskite oxides for oxygen evolution reaction by combining experimental and ab initio studies. ACS Catal. 5, 4337-4344 (2015)

\section{Acknowledgements}

This work was supported by Basic Science Research Program through the National Research Foundation of Korea (NRF) grant funded by the Korea government (MSIT) (No. 2019R1A2C1009025, 2019R1A4A1029237, and 2020R1A4A3079710). Y.-K.H would like to thank Bohyun Hwang and Sujin Lee for their technical support.

\section{Author contributions}

N.K.O. carried out most of the experimental work and wrote the manuscript. J.S. wrote the manuscript and contributed to the designing of schematics. S.L. and H.-J.K. performed the DFT calculation and wrote the manuscript. U.K. helped the interpretation of electrochemical analysis. J.L. performed XPS measurements. Y.-K.H. directed the theoretical work H.P. conceived the project and directed the overall work and manuscript writing. All the authors contributed to the discussion and analysis of the results regarding the manuscript.

\section{Competing interests}

The authors declare no competing interests. 


\section{Additional information}

Supplementary information The online version contains supplementary material available at https://doi.org/10.1038/s41467-021-24829-8.

Correspondence and requests for materials should be addressed to Y.-K.H. or H.P.

Peer review information Nature Communications thanks Vera Butova and other, anonymous, reviewers for their contributions to the peer review of this work. Peer review reports are available.

Reprints and permission information is available at http://www.nature.com/reprints

Publisher's note Springer Nature remains neutral with regard to jurisdictional claims in published maps and institutional affiliations. (c) (i) Open Access This article is licensed under a Creative Commons Attribution 4.0 International License, which permits use, sharing, adaptation, distribution and reproduction in any medium or format, as long as you give appropriate credit to the original author(s) and the source, provide a link to the Creative Commons license, and indicate if changes were made. The images or other third party material in this article are included in the article's Creative Commons license, unless indicated otherwise in a credit line to the material. If material is not included in the article's Creative Commons license and your intended use is not permitted by statutory regulation or exceeds the permitted use, you will need to obtain permission directly from the copyright holder. To view a copy of this license, visit http://creativecommons.org/ licenses/by/4.0/.

(C) The Author(s) 2021 